\title{
Gamma irradiation of graphene quantum dots with ethylenediamine: Antioxidant for ion sensing
}

\author{
Svetlana Jovanovića, ${ }^{\mathrm{a}, *}$, Slađana Dorontića ${ }^{\text {, Dragana Jovanovića }}{ }^{\mathrm{a}}$, Gabriele Ciasca ${ }^{\mathrm{b}}$, Milica Budimir ${ }^{\mathrm{a}}$, \\ Aurelio Bonasera ${ }^{\mathrm{c}}$, Michelangelo Scopelliti ${ }^{\mathrm{c}}$, Olivera Marković ${ }^{\mathrm{d}}$, Biljana Todorović Markovića ${ }^{\mathrm{a}}$ \\ a "Vinča” Institute of Nuclear Sciences - National Institute of the Republic of Serbia, University of Belgrade, P. O. Box 522, 11001, Belgrade, Serbia \\ ${ }^{\mathrm{b}}$ Istituti Biologici-Istituto di Fisica, Università Cattolica del Sacro Cuore, Largo Francesco Vito 1, 00168, Roma, Italy \\ ${ }^{\mathrm{c}}$ Dept. of Physics and Chemistry, Emilio Segrè, University of Palermo and Consorzio Interuniversitario Nazionale per la Scienza e Tecnologia dei Materiali (INSTM), \\ Palermo Research Unit, Viale delle Scienze, Bdg. 17, 90128, Palermo, Italy \\ ${ }^{\mathrm{d}}$ Department of Chemistry, IChTM, University of Belgrade, Njegoševa 12, 11000, Belgrade, Serbia
}

\section{A R T I C L E I N F O}

Presented results indicate that in the selected medium, gamma irradiation at low doses induces the lowering in the GQDs height due to the lower number of graphene layers in their structure. Unexpectedly, irradiation in the same medium at $200 \mathrm{kGy}$ induce a significant increase in GQDs height and caused observable agglomeration.

Keywords:

Carbon

Chemical properties

Optical properties

Sensor

\begin{abstract}
A B S T R A C T
Due to the low consumption of chemicals, the absence of toxic residual side products, the procedure simplicity and time-saving aspects, gamma irradiation offers advantages over the classical chemical protocols. We successfully employed gamma irradiation in order to introduce N-atoms in Graphene Quantum Dots (GQDs). By irradiating GQDs water dispersions in the presence of isopropyl alcohol and ethylenediamine, at doses of 25, 50 and $200 \mathrm{kGy}$, we attached amino groups onto GQDs in a single synthetic step. At the same time, a chemical reduction is achieved, too. Selected conditions induced incorporation of N-atoms within GDQs atomic lattice (around 3 at\%), at all applied doses. Additionally, the C-atoms percentage was highly increased, from 63 to 79 at $\%$ or higher. The zeta potential of dots changed from -34.6 to $+9.1 \mathrm{mV}$, due to the modification of functionalizing groups localized at the surface. Produced chemical changes lead to the desired alteration of the GQDs optical properties, such as an increased photoluminescence intensity, a higher photoluminescence quantum yields (from 2.07 to $18.40 \%$ ) and a narrowing of the spectral features in the emission spectra. The ability of gamma-irradiated GQDs to quench free radical species was investigated and positively assessed; additionally, non-enzymatic optical detection of $\mathrm{Cu}(\mathrm{II})$ ions using GQDs as a sensor was studied and the detection limits are herein reported. These results suggest that GQDs can be potentially applied as smart photoluminescent sensors for metal cations.
\end{abstract}

\section{Introduction}

Graphene Quantum Dots (GQDs) are zero-dimensional carbon-based nanomaterial. They were discovered in 2008. by Ponomarenko et al. [1] Both graphene sheets and oxygen-containing functional groups are typically present in the GQDs structure [1,2]. Graphene constitutes the core component of the dots, while oxygen-containing functional groups are located on the basal plane (epoxy or hydroxyl groups) or at the edges (carboxyl or carbonyl groups) [3-5]. Depending on the number of graphene layers, dots can be defined as single GQDs-with one graphene layer, double- or few-GDQs with more graphene layers [6]. The lateral size of GQDs is usually lower than $100 \mathrm{~nm}$ and the height is in the range 0.4-3 nm, depending on the graphene layers number [7].

Oxygen-containing functional groups are responsible for good solubility in polar solvents such as water or ethanol $[7,8]$ in the concentration of $5 \mathrm{mg} \mathrm{mL}^{-1}$ [9] or even higher, $>15 \mathrm{mg} \mathrm{mL}^{-1}$ [10]. Properties that distinguish GQDs from other carbon nanomaterials such as carbon nanotubes and graphene, are high dispersibility in water and polar organic solvents, photoluminescence [11], good biocompatibility [12]. In comparison with conventional inorganic quantum dots, GQDs are less toxic and more resistive to photobleaching under prolonged light irradiation with respect to luminescent organic dyes [13]. Moreover, GQDs show intense optical absorption in the UV region, with Photoluminescence (PL) usually emitted in the visible part of the spectrum [14].

Due to these properties, GQDs have been investigated for possible applications in sensing [14-17]. As fluorescent probes, GQDs were investigated for the detection of metal ions, pesticides or other organic species [14]. Scientific research is funneling huge resource to optimize and improve sensing methodologies. Environmental chemistry urges

\footnotetext{
* Corresponding author. Vinča Institute of Nuclear Sciences, P. O. Box 522, 11001, Belgrade, Serbia.

E-mail address: svetlanajovanovic@vinca.rs (S. Jovanović).
} 
new sensitive and low-cost approaches for the determination of toxic species or dangerous analytes in different matrices, ranging from soil samples, liquid aqueous waste and air samples collected in urban areas [18-20]. Food analysis is another field where it is extremely valuable the possibility to assess the presence of toxic molecules, or species intimately related to the undergoing spoilage processes [21,22]. Amines are one of the most well-known examples of biomolecules whose presence and concentration is related to the quality of food matrices [23]; several colorimetric strategies have been reported and reviewed in the literature, based on the evolution of either light absorption [24-26] or emission features [27,28]. In the case of optical sensing, information can be retrieved focusing on GQDs absorbance and fluorescent emissions; thus, modification of absorbance intensity, or fluorescence quenching efficiency is a well-assessed tool for revealing an analyte, as a result of interaction between GQDs probe and investigated species [16]. In contrast to different methods for the detection of metal ions, such as atomic absorption, plasma-mass and plasma-atomic emission spectroscopies, the fluorescent probes with GQDs have higher sensitivity and selectivity [29]. Other advantages of this approach are dispersibility in aqueous solution, low-cost, good reproducibility and biocompatibility [16].

The ability of GQDs to detect Fe(III) ions by quenching of photoluminescence has been reported by Zhou et al. [30] A polycyclic aromatic hydrocarbon (PAH), such as pyrene, benzo[a]pyrene, or naphtho [2,3-1]pyrene) under acidic conditions and hydrothermal reduction with hydrazine hydrate was used for GQD production. Fe(III) ions show selective affinity to quenching GQDs photoluminescence, with a detection limit as low as $5 \cdot 10^{-9} \mathrm{M}$. Also, the redox couple $\mathrm{Fe}(\mathrm{II}) / \mathrm{Fe}(\mathrm{III})$ may be used for the detection of oxidant species such as hydrogen peroxide [30]. Pesticides are another example of the environmentally relevant analyte to be assessed, which can also be detected by GQDs [31]. Roushani et al. have developed a dual photoluminescent sensor for the detection of both $\mathrm{Hg}$ (II) ions and Malathion (2-(dimethoxyphosphinothioylthio) butanedioic acid diethyl ester), which is widely applied in the agriculture as an insecticide [31]. GQDs were synthesized using a bottom-up method, pyrolyzing citric acid at $190{ }^{\circ} \mathrm{C}$ under atmospheric pressure. It was observed that the PL of GQDs was quenched by mercury ions and restored by the addition of Malathion. These PL intensity variations showed acceptable linearity for both analytes in the concentration ranges $1-10 \mu \mathrm{M}$ and the limit of detection (LOD) for Malathion was $0.5 \mu \mathrm{M}\left(\mathrm{R}^{2}=0.99\right)$ [31].

To explore the potential use of GQDs as sensors in PL-based detection methodologies, it is desirable to improve their optical properties such as PL intensity and quantum yield. Among different strategies to achieve improved PL features, the heteroatoms incorporation within the GQD structure is broadly investigated [32-35]. There are already several reports in the literature where S, N, B, P, Se, and other atoms were incorporated within carbon-based quantum dots during GQDs synthesis [36-40].

Among graphene-based nanomaterials used as a pristine scaffold for the synthesis of GDQs, graphene oxide (GO) is one of the most reported. In a recent report, it was irradiated in the presence of ethylenediamine (EDA) and the incorporation of $\mathrm{N}$ atoms was observed [41]. By irradiating a GO water dispersion, in the presence of $3 \mathrm{vol} \%$ of EDA with doses of 5.3, 15, 20 and $35.3 \mathrm{kGy}$, Li et al. observed the elimination of the hydroxyl, epoxide and carboxyl groups as well as incorporation $\mathrm{N}$ atoms in the GO structure. The highest percentage of $\mathrm{N}$-atoms incorporation was achieved under samples irradiation at a dose of $35 \mathrm{kGy}$, and it was 10.44 at\% [41]. Another study showed that GO irradiated at a dose of $25 \mathrm{kGy}$, in water/ethanol mixture with alkylamine of various lengths such as octadecylamine, or dodecylamine, tetradecylamine and hexadecylamine, leads to chemical reduction as well as attaching of N-functional groups in the GO structure [42].

In this paper, we document the PL intensity increase of GQDs by introducing $\mathrm{N}$-atoms in their structure by using a specifically designed protocol based on the gamma irradiation of pristine GDQs in the presence of EDA. We applied doses of 25, 50 and $200 \mathrm{kGy}$ on the base of our previously reported approach, which proved significant improvement of PL intensity and chemical reduction at these doses $[11,13,43]$. To explore the possible applications of new GQDs, we analyzed antioxidative properties and variations in emitted PL spectra in the presence of $\mathrm{Cu}(\mathrm{II})$ ions. The ability of gamma-irradiated GQDs to quench free radicals was investigated using the DPPH assay, Experimental section [44]. Additionally, modified GQDs were investigated as a fluorescent probe for the detection of $\mathrm{Cu}(\mathrm{II})$ ions.

\section{Experimental}

\subsection{Synthesis of pristine and modified GQDs}

For the synthesis of pristine GQDs, we used previously described, electrochemical top-down procedure [45]. The starting material consisted of the spectroscopic graphite electrode purchased from Ringsdorff Werke GmbH (Bonn, Germany), 99.999\% purity and $\emptyset=3.05 \mathrm{~mm}$. First, electrodes were thoroughly washed with MiliQ water and ethanol. $3 \mathrm{~g}$ of $\mathrm{NaOH}$ was dispersed in ethanol ( $96 \mathrm{vol} \%)$ and was used as the electrolyte. Then, the electrodes were immersed in the dispersion. Graphite electrodes were used both as cathode and anode. The current was set at $20 \mathrm{~mA}$, while the applied potential was $20 \mathrm{~V}$. After $24 \mathrm{~h}$, the electrolyte changed color from pale yellow to dark brown. In order to isolate GQDs, we first evaporated ethanol under reduced pressure, then the crude material was redispersed in water. We used MWCO $3.5 \mathrm{kDa}$ dialysis bags in order to remove the residual $\mathrm{NaOH}$ from GQDs. The $\mathrm{pH}$ value of the solution was constantly monitored and, when $\mathrm{pH}$ reached a value of 7 , water was changed one more time and the dispersion was collected the next day. After dialysis, water was evaporated from GQDs dispersion and the powder was collected. These dots are labeled in the paper as pristine Graphene Quantum Dots (p-GQDs).

Gamma irradiation was used to modify the GQDs structure. The reaction medium consisted of a mixture of 4 vol\% EDA, 3 vol\% isopropyl alcohol (IPA) and GQDs in ultrapure water in the final concentration of $1 \mathrm{mg} \mathrm{mL}^{-1}$. Before irradiation, samples were sonicated while bubbling with Ar in order to remove molecular oxygen from the dispersion. Then, these mixtures were irradiated using irradiation units with Co-60 as an irradiation source. Applied doses were 25, 50 and $200 \mathrm{kGy}$. After irradiation, GQDs were isolated from the irradiation medium by dialysis and the resulting water-dispersions of gamma-irradiated GQDs were dried. Collected powders were used for further analysis. Obtained samples irradiated with doses of 25, 50 and $200 \mathrm{kGy}$ were named GQDs-25-EDA, GQD-50-EDA and GQDs-200-EDA, respectively.

\subsection{Methods}

Absorption measurements were performed at a Shimadzu UV-2600 UV-Visible spectrophotometer (Shimadzu Corporation, Tokyo, Japan) in the 200-800 $\mathrm{nm}$ range with $1 \mathrm{~nm}$ step. The GQD powders were dispersed in ultrapure water in a concentration of $0.25 \mathrm{mg} \mathrm{mL}^{-1}$ and recorded at $20{ }^{\circ} \mathrm{C}$ under a normal atmosphere.

The PL measurements of the samples were conducted on Horiba Jobin Yvon Fluoromax-4 spectrometer (Horiba, Kyoto, Japan), equipped with Peltier element and magnetic stirrer for cuvette, using quartz cell with $1 \mathrm{~cm}$ path length and $4 \mathrm{~mL}$ volume. Excitation wavelengths were in the 300-400 $\mathrm{nm}$ range. The GQD samples were dispersed in methanol, in a concentration of $0.25 \mathrm{mg} \mathrm{mL}^{-1}$. The spectra were collected in the air environment at a temperature of $25{ }^{\circ} \mathrm{C}$ in the quartz cuvette. Fluorescence quantum yields (QY) were estimated by integrating the area under the fluorescence curves using the equation below [46]:

$\mathrm{QY}_{\mathrm{GQDs}}=\mathrm{QY}_{\mathrm{REF}}\left(\mathrm{A}_{\mathrm{REF}} / \mathrm{A}_{\mathrm{GQDs}}\right)\left(\mathrm{F}_{\mathrm{GQDs}} / \mathrm{F}_{\mathrm{REF}}\right)\left(\mathrm{n}_{\mathrm{GQDs}} / \mathrm{n}_{\mathrm{REF}}\right)^{2}$

where $Q Y$ is fluorescence quantum yield, $F$ is integrated fluorescence 
intensity, $A$ is the absorbance values, $n$ is the refractive index of the solvent, while subscripts 'REF' and 'GQDs' refer to the used reference (Rhodamine B, QY $=31 \%$ ) and GQDs samples [46,47].

Atomic Force Microscopy (AFM) measurements were performed using Quesant (Agoura Hills, CA, Unites States) microscope operating in tapping mode in the air, at room temperature. We used the Q-WM300 AFM probe, rotated, monolithic silicon probe for non-contact highfrequency applications. Standard silicon tips (NanoAndMore Gmbh, Wetzlar, Germany) were used, with a force constant of $40 \mathrm{~N} / \mathrm{m}$. For the preparation of sample surfaces, GQDs were dispersed in MiliQ water in a concentration of $0.25 \mathrm{mg} \mathrm{mL}^{-1}$. Dispersions were spin-coated on mica which was used as the substrate. For AFM images analysis, Gwyddion 2.53 software was used [48].

Transmission Electron Microscope (TEM) JEOL JEM-2100F (JEOL, Ltd., Tokyo, Japan) was used to analyze GQDs structure and morphology. Powder samples of GQDs were dispersed in water in a concentration of $1 \mathrm{mg} \mathrm{mL}^{-1}$ and deposited on carbon laced copper grids. The lateral size of GQDs and interlayer distance was measured from TEM images using ImageJ software.

Dynamic Light Scattering (DLS) and $\zeta$-Potential measurements were performed with a Zetasizer Nano ZS instrument (Malvern, Herrenberg, Germany) equipped with a 633-nm He-Ne laser and operating at an angle of $173^{\circ}$. Measurements were performed at a fixed position $(4.65 \mathrm{~mm})$ with an automatic attenuator and at a controlled temperature $\left(20^{\circ} \mathrm{C}\right)$. For each sample, ten measurements were averaged and the number weighted size distribution of the particles was retrieved. Solvent-resistant micro cuvettes (ZENO040, Malvern, Herrenberg, Germany) have been used for experiments with a sample volume of $40 \mu \mathrm{L}$. DLS measures the intensity based on the autocorrelation function:

$\mathrm{G}_{2}(\tau)=\langle\mathrm{I}(\mathrm{t}+\tau) \mathrm{I}(\mathrm{t})\rangle /\langle\mathrm{I}\rangle^{2}$

where $\tau$ is the lag time and the brackets represent the ensemble average. The $\mathrm{G}_{2}(\tau)$ can be related to the field autocorrelation function $g_{1}(t)$ through the Siegert relation $G_{2}(\tau)=1+\beta\left|g_{1}(t)\right|^{2}$, where $\beta$ is an instrumental constant equal to 1 in our setup. $\zeta$-potential of $\mathrm{p}$ - and gamma-irradiated GDQs was calculated from the electrophoretic mobility by means of the Henry correction to Smoluchowski's equation, implemented in the Z-sizer data-analysis software. To this purpose 5 measurements were averaged.

Fourier Transform-InfraRed (FTIR) spectra were obtained in attenuated total reflection (ATR) mode on the Thermo Scientific Nicolet 6700 FTIR instrument (Thermo Fischer Scientific, Waltham, MA, United States). The spectral resolution was $2 \mathrm{~cm}^{-1}$. GQD in the form of powders were used for FTIR measurements.

X-ray Photoelectron Spectroscopy (XPS) was acquired by using a ULVAC-PHI PHI500 VersaProbe II scanning microprobe (ULVAC-PHI, Inc., Chigasaki, Japan), with an $\mathrm{Al} \mathrm{K \alpha}$ source (1486.6 eV), $100 \mu \mathrm{m}$ spot, $25 \mathrm{~W}$ power, $15 \mathrm{kV}$ acceleration and $45^{\circ}$ take-off angle. All spectra were collected using a dual neutralization system (both $\mathrm{e}^{-}$and $\mathrm{Ar}^{+}$).

Radical scavenging activity (RSA) was also investigated. The molecule of 2,2-diphenyl-1-picrylhydrazyl (DPPH) was used to investigate the antioxidative activity of pristine and gamma-irradiated GQDs. First, DPPH, in a concentration of $120 \mu \mathrm{M}$ in methanol, was added to GQDs dispersions. GQDs were also dispersed in methanol. The concentrations of GQD dispersions ranged $2.5-200 \mu \mathrm{g} / \mathrm{ml}$ while the amount of DPPH was kept at the same level in each mixture. Both DPPH and GQDs samples were mixed and then absorption for $1 \mathrm{~h}$. After incubation, UV-Vis spectra of each mixture were measured by recording the intensity of absorbance in the range of $350-800 \mathrm{~nm}$. The molecule of DPPH is a stable radical with a maximum of absorption at $515 \mathrm{~nm}$ [49]. When DPPH radicals react with antioxidants, these radicals were quenched which can be observed by color changing from violet to yellow as well as the intensity lowering of the absorption band at $515 \mathrm{~nm}$ in UV-Vis spectra. The absorbance was measured at a Shimadzu
UV-2600 UV-Visible spectrophotometer (Shimadzu Corporation, Tokyo, Japan). For comparison, we used ascorbic acid methanol solutions, at $10-200 \mu \mathrm{M}$ concentration range. The radical scavenging activity (RSA) was calculated according to the formula:

$\operatorname{RSA}(\%)=\left(\mathrm{A}_{\mathrm{c}}-\mathrm{A}_{\mathrm{GQDs}}\right) / \mathrm{A}_{\mathrm{c}} \cdot 100$

where $A_{c}$ is the intensity of absorption of control (DPPH in methanol) and $A_{G Q D}$ is the intensity of the absorption band of a mixture of GQDs with DPPH also in methanol. All measurements were repeated three times.

Fluorescence quenching with different concentrations of $\mathrm{Cu}(\mathrm{II})$ ions was studied. The total concentration of GQDs dispersed in ultrapure water was $0.03 \mathrm{mg} \mathrm{mL}^{-1}$. Different volumes of the $\mathrm{Cu}(\mathrm{II})$ solution were added to the prepared dispersion in order to adjust the final measured concentrations $\left(0-80.5 \mu \mathrm{mol} \mathrm{L}^{-1}\right)$. Before every measurement, the mixture of solutions was incubated for $5 \mathrm{~min}$ at room temperature. For each concentration of $\mathrm{Cu}(\mathrm{II})$ ions, measurements were repeated three times. The fluorescence emission spectra were recorded using a Horiba Jobin Yvon Fluoromax-4 spectrometer under $360 \mathrm{~nm}$ excitation wavelength.

\section{Results and discussion}

\subsection{UV-Vis spectroscopy}

To investigate the optical properties of GQDs, we used UV-Vis spectroscopy and the obtained absorption spectra are presented in Fig. 1. For all samples, the high-intensity absorption is observed at lower wavelengths, and decreases exponentially with the increase of wavelength. The main absorption band in the spectrum of p-GQDs is centered at around $220 \mathrm{~nm}$ and it stems from $\pi-\pi^{*}$ transitions of aromatic C-C bonds. This band is also observed in samples of GQDs-25EDA, GQDs-50-EDA, and GQDs-200-EDA and it is shifted to the lower wavelength for all the three samples (around $200 \mathrm{~nm}$ ). This shift could be attributed to the changes in the prevalence of aromatic bonds. The band at $365 \mathrm{~nm}$ which stems from $\mathrm{n}-\pi^{*}$ transitions in $\mathrm{C}=\mathrm{O}$ bonds is observed in the spectrum of p-GQDs. Additionally, similar shoulder bands are observed more clearly in gamma-irradiated samples, located at $248 \mathrm{~nm}$ for GQD-25-EDA, $249 \mathrm{~nm}$ for GQDs-50-EDA and $270 \mathrm{~nm}$ in the spectrum of GQDs-200-EDA. These bands origin from carbonyl functional groups and shifts in their positions can also be an indication of changes in the chemical environment of these functional groups. Thus, the chemical composition of each GQD sample has to be

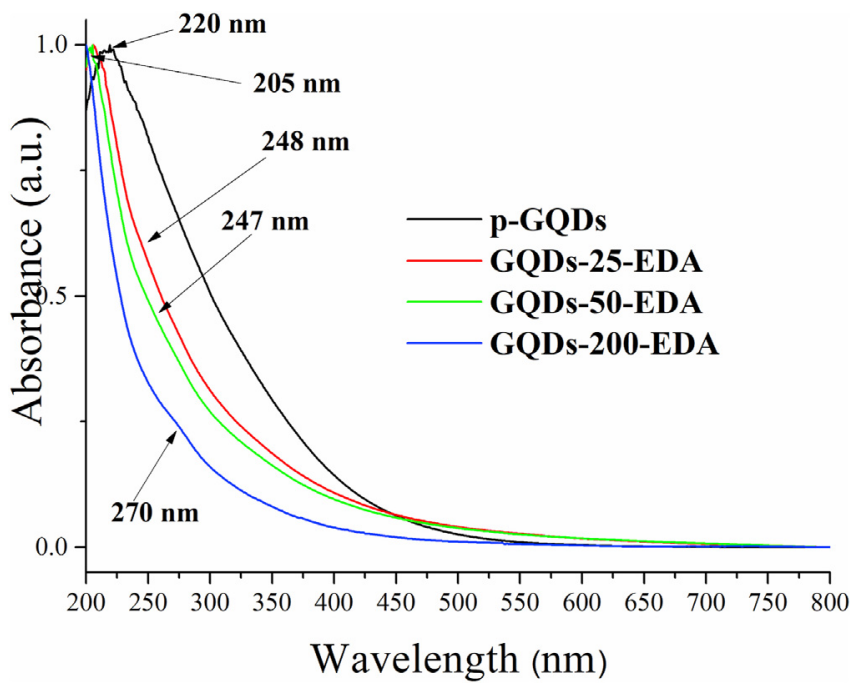

Fig. 1. UV-Vis spectra of p-GQDs (black curve), GQDs-25-EDA (red curve), GQDs-50-EDA (green curve) and GQDs-200-EDA (blue curve). 

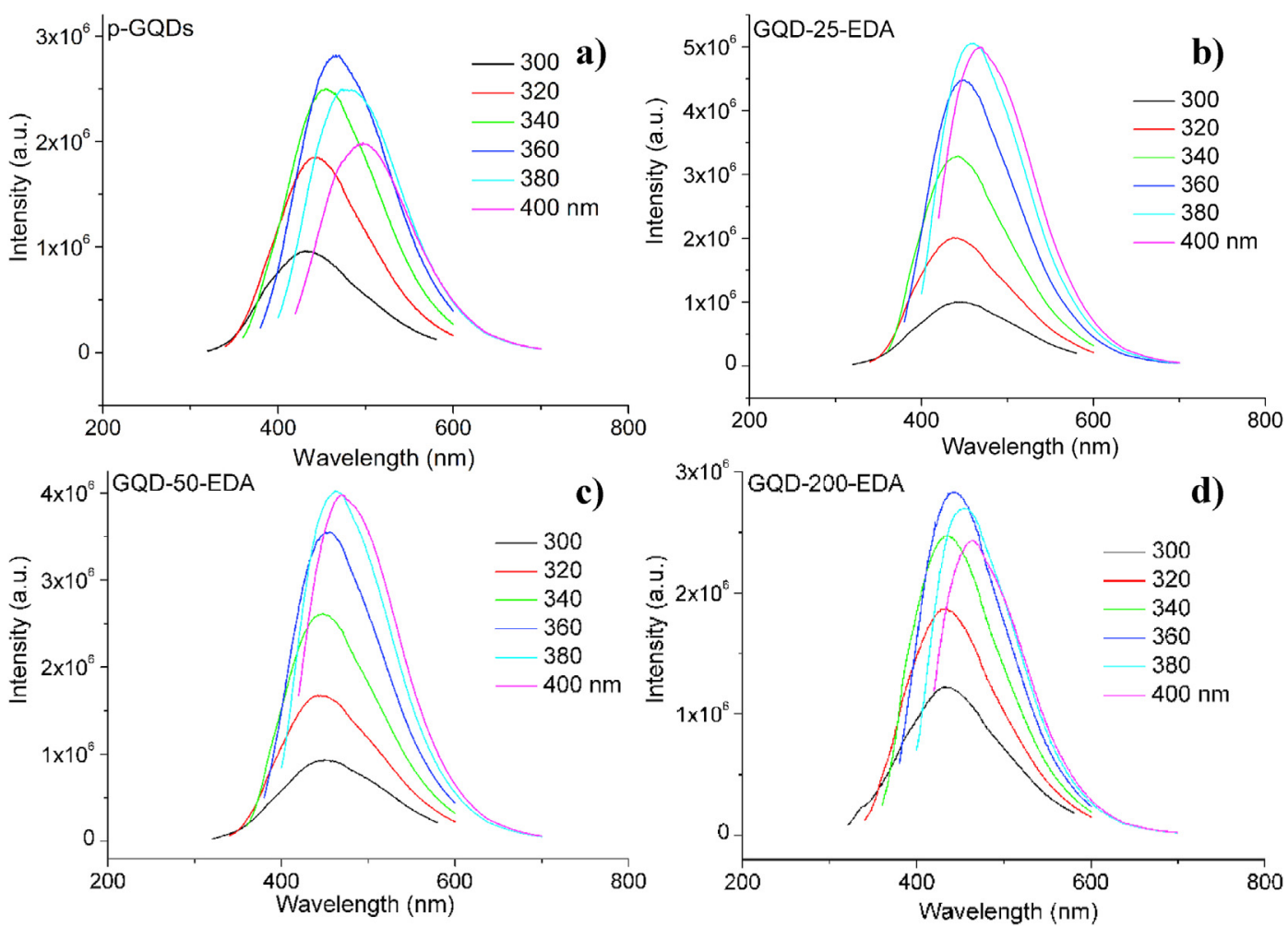

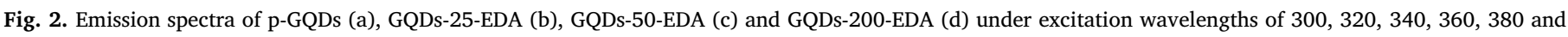
$400 \mathrm{~nm}$.

investigated.

\subsection{Photoluminescence spectroscopy}

The fluorescence emission spectra of modified GQDs under different excitation wavelengths in the range of $300-400 \mathrm{~nm}$ are presented in Fig. 2. To closely investigate PL properties of GQDs, the position, FWHM and the intensities of each band are listed in Table 1.

As it can be observed in all the spectra, at different excitation wavelengths, the maximum of the emission band is shifted. This kind of behavior is called excitation-dependent PL and it is often observed in samples of GQDs [50-52]. Although a great scientific effort was invested in finding a reason for this GQDs property, there is still a lot of discussion in order to better elucidate the mechanism behind this phenomenon [50]. Different models explaining the PL behavior of GQDs coexist in the literature: size-dependent quantum confinement effects [53], surface trap states determined by functional groups [54], armchair vs. zigzag configuration of edges [55], the electronegativity of heteroatoms [56,57]. Each model explains multicolor, excitation-depended PL emission at some level.

Herein, it can be observed that GQDs irradiated in the presence of EDA showed a less pronounced excitation-dependent behavior: in the case of p-GQDs, the emission bands shifted from 430 to $490 \mathrm{~nm}$, while in the case of all gamma-irradiated samples, these shifts are about $25 \mathrm{~nm}$ in magnitude, for example, 445-468 nm (GQD-25-EDA), $450-473 \mathrm{~nm}$ for GQDs-50-EDA and 431-461 nm for a dose of $200 \mathrm{kGy}$. These results show that synthesized GQDs emit violet to blue and even green light, while after gamma irradiation, dots emit mostly blue light. Several research reports indicated that blue photoluminescence of GQDs stems from $\mathrm{sp}^{2}$ core, while green originates from functional groups [58,59]. Other studies proved that carboxyl and amide edge groups are responsible for the efficient green emission and that the blue emission results from the presence of hydroxyl groups [60,61]. Thus, before discussing PL origin and excitation-dependent PL emission, both the size and chemical composition, including the level of heteroatoms
Table 1

Data retrieved by recording wavelength-dependent light emission spectra for pGDQs and the gamma-irradiated samples. The position of emission maximum at excitation wavelengths indicated as a subscript, FWHM, and the intensity of observed bands.

\begin{tabular}{|c|c|c|c|}
\hline Sample & Emission position/nm & FWHM/nm & Intensity/cps \\
\hline \multirow[t]{6}{*}{ p-GQDs } & $430^{\mathrm{Ex} 300}$ & 127 & 903749 \\
\hline & $442^{\mathrm{Ex} 320}$ & 116 & 1847283 \\
\hline & $455^{\mathrm{Ex} 340}$ & 115 & 2501273 \\
\hline & $466^{\mathrm{Ex} 360}$ & 114 & 2807654 \\
\hline & $478^{\mathrm{Ex} 380}$ & 131 & 2488539 \\
\hline & $495^{\mathrm{Ex}} 400$ & 120 & 1976938 \\
\hline \multirow[t]{6}{*}{ GQD-25-EDA } & $445^{\text {Ex } 300}$ & 150 & 995415 \\
\hline & $440^{\text {Ex } 320}$ & 120 & 2000990 \\
\hline & $442^{\text {Ex } 340}$ & 110 & 3283672 \\
\hline & $448^{\text {Ex } 360}$ & 121 & 4466185 \\
\hline & $460^{\text {Ex } 380}$ & 121 & 5063667 \\
\hline & $468^{\text {Ex } 400}$ & 131 & 5019524 \\
\hline \multirow[t]{6}{*}{ GQD-50-EDA } & $450^{\text {Ex } 300}$ & 160 & 933955 \\
\hline & $445^{\text {Ex } 320}$ & 129 & 1681550 \\
\hline & $446^{\text {Ex } 340}$ & 116 & 2613572 \\
\hline & 451 Ex 360 & 109 & 3552914 \\
\hline & $462^{\text {Ex } 380}$ & 121 & 4027447 \\
\hline & $473^{\text {Ex } 400}$ & 130 & 3990662 \\
\hline \multirow[t]{6}{*}{ GQD-200-EDA } & 431 Ex 300 & 127 & 1221731 \\
\hline & 430 Ex 320 & 116 & 1859705 \\
\hline & $433^{\text {Ex } 340}$ & 111 & 2470557 \\
\hline & $442^{\text {Ex } 360}$ & 109 & 2839841 \\
\hline & $455^{\text {Ex } 380}$ & 120 & 2700198 \\
\hline & $463^{\mathrm{Ex} 400}$ & 130 & 2436757 \\
\hline
\end{tabular}

in GQDs must be assessed in detail.

The intensity of PL is varying with excitation wavelengths, as well. In the case of p-GQDs, the highest intensity is observed when GQDs were excited with light at $360 \mathrm{~nm}$, which is the same as in the case of GQDs-200-EDA (Fig. 2, d). In the case of GQDs irradiated at 25 and $50 \mathrm{kGy}$, the highest intensities in emission spectra were observed under 
Table 2

The values of intensities of emission and adsorption bands and relative QY obtained at two excitation wavelengths.

\begin{tabular}{|c|c|c|c|c|c|c|}
\hline Sample & $\mathrm{I}_{\mathrm{ex} 320}$ & $\mathrm{I}_{\mathrm{ex} 340}$ & $\mathrm{~A}_{320}$ & $\mathrm{~A}_{340}$ & $\mathrm{QY}_{320}(\%)$ & $\mathrm{QY}_{340}(\%)$ \\
\hline p-GQDs & 24874229 & 23450791 & 0.177 & 0.144 & 2.07 & 1.45 \\
\hline GQDs-25-EDA & 65230867 & 83253437 & 0.198 & 0.165 & 5.11 & 4.69 \\
\hline GQDs-50-EDA & 23037085 & 27649752 & 0.190 & 0.162 & 1.87 & 1.59 \\
\hline GQDs-200-EDA & 48028778 & 59680118 & 0.198 & 0.166 & 18.40 & 16.61 \\
\hline Rhodamin B & 298523499 & 423235189 & 0.148 & 0.122 & 31 & 31 \\
\hline
\end{tabular}

380 and $400 \mathrm{~nm}$ excitation wavelengths (Table 1).

The changes can also be noticed in the values of full width at halfmaximum (FWHM) of emission bands (Table 1). Upon excitation, at 340 and $360 \mathrm{~nm}$ the emission bands are the narrowest for p-GQDs. In the case of gamma-irradiated samples, these values are a bit lower (109 csp compared to 115 for p-GQDs).

Relative Quantum Yield (QY) of photoluminescence was calculated using Rhodamine B as a reference. In Table 2, the values of QY obtained at two different excitation wavelengths are presented. The largest increase in PL QY is noticed for GQDs-200-EDA, 18.40 at the excitation wavelength of $320 \mathrm{~nm}$.

Analysis of PL properties showed that gamma irradiation in selected condition induced significant changes in optical properties:

- a less pronounced excitation-depended behavior, emission peak shifts of about $30 \mathrm{~nm}$ under the excitation from 300 to $400 \mathrm{~nm}$;

- a small reduction in the bandwidth (FWHM);

- higher intensity of emission bands;

- significantly higher PL QYs.

\subsection{AFM analysis}

AFM images (Fig. 3) reveal that p-GQDs, GQDs-25-EDA and GQDs50-EDA are highly dispersed in water with a typical topographic height between 0.5 and $2 \mathrm{~nm}$. The height histograms presented in each AFM image indicates that most GQDs consist of ca. 1-4 graphene layers [62]. Compared to p-GQDs, both GQDs-25-EDA and GQDs-50-EDA samples have larger percentages of single-layer GQDs. In the case of GQDs-25EDA, the low-height fraction of GQDs is the most abundant fraction. These results suggest that gamma irradiation in this specific experimental setup increases the level of layer separation and thus, increases the dispersibility of GQDs in water.

On the contrary, irradiation at $200 \mathrm{kGy}$ induces the formation of large agglomerates with a height of around $10-20 \mathrm{~nm}$ (Fig. 3d). As can be observed, the lateral size of particles is much larger compared to other samples (up to $200 \mathrm{~nm}$ ), while in other AFM images lateral size is below $50 \mathrm{~nm}$. Additionally, the particles in agglomerates can be observed and their size is in the range of $30 \mathrm{~nm}$.
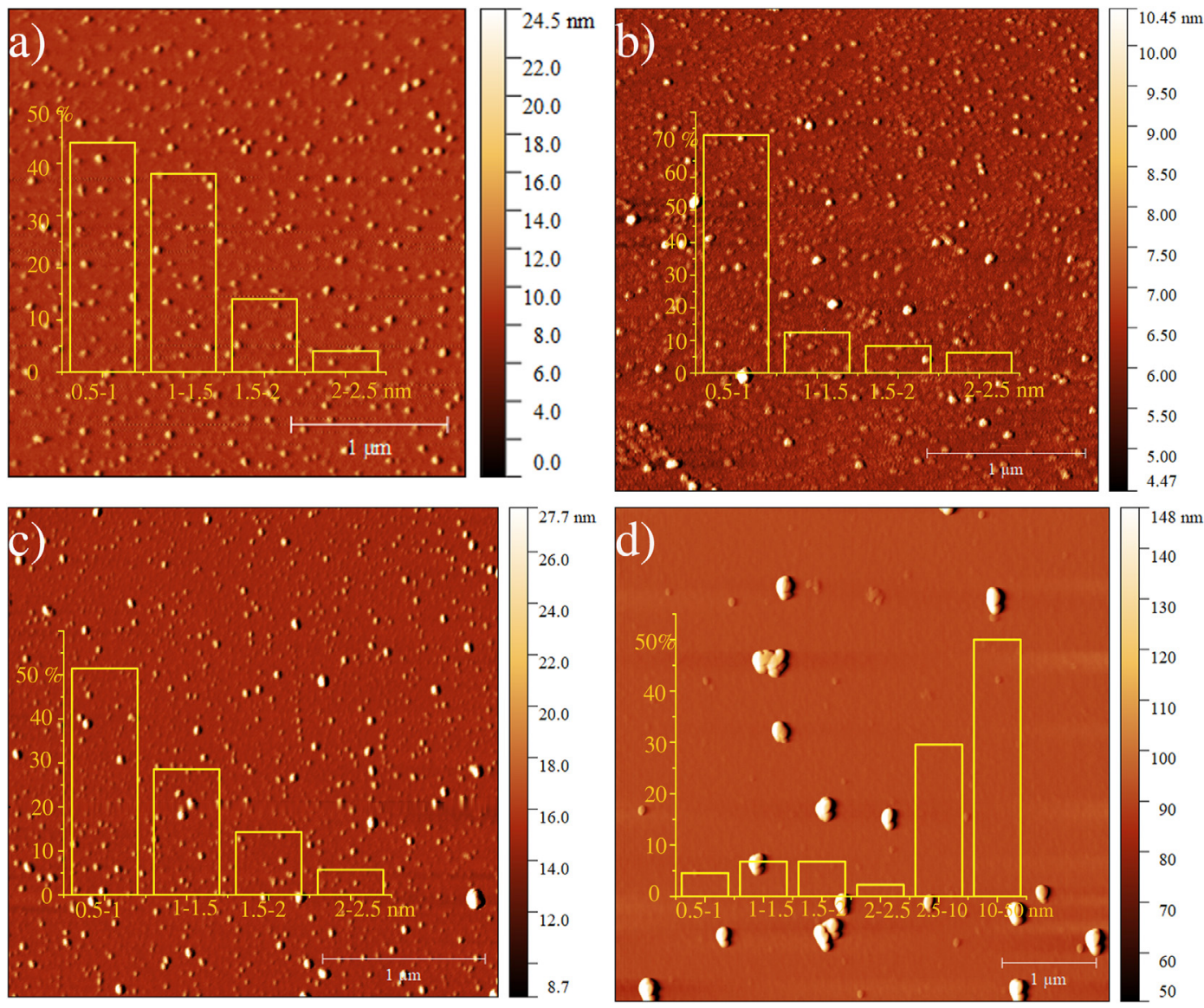

Fig. 3. AFM images and histograms of the height distributions of p-GQDs (a), GQDs-25-EDA (b), GQDs-50-EDA (c) and GQDs-200-EDA (d). 

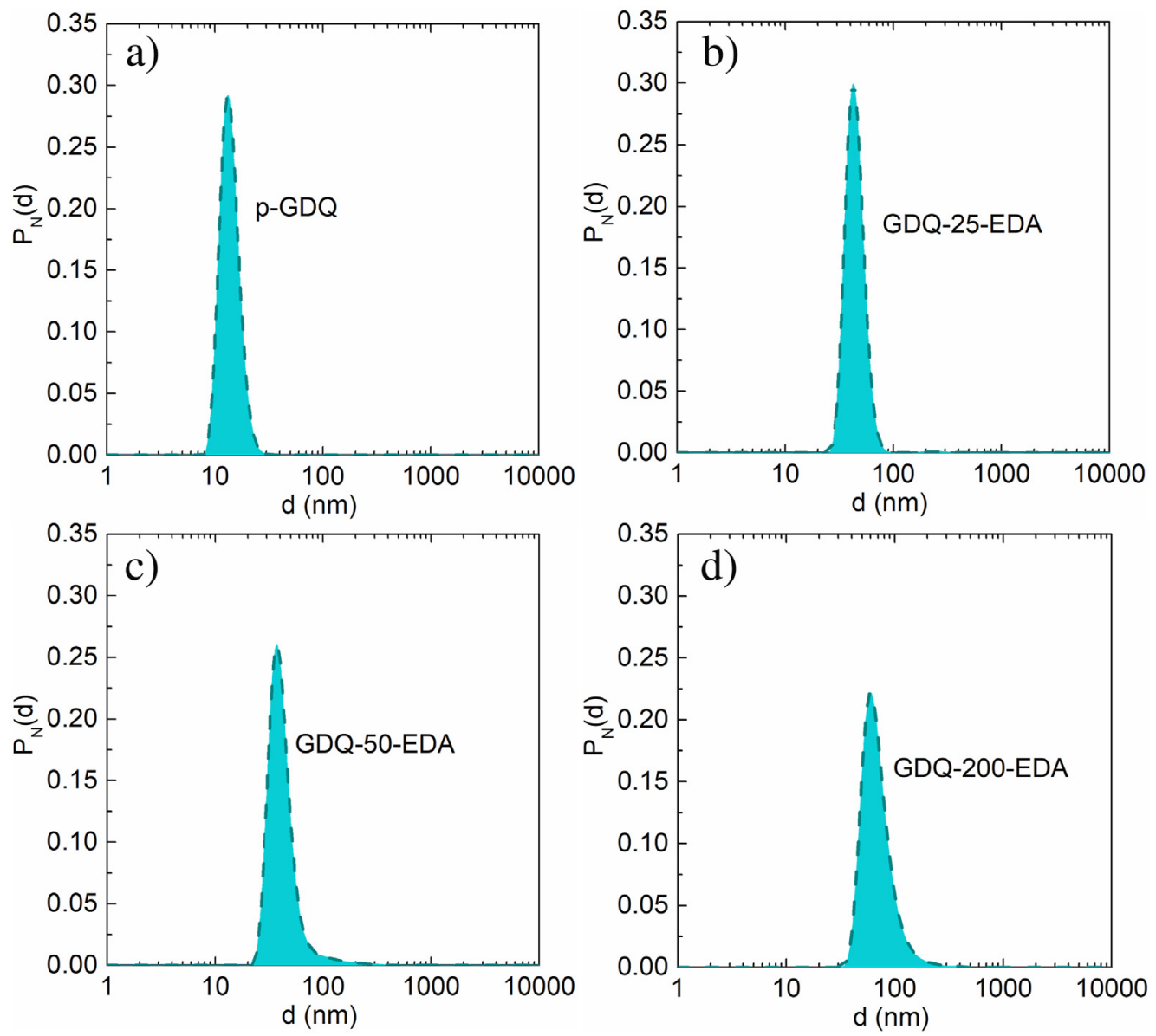

Fig. 4. DLS diameter distribution for p-GQDs (a), GQDs-25-EDA (b), GQDs-50-EDA (c) and GQDs-200-EDA (d).

\subsection{DLS and zeta potential measurement}

The size distribution of GQDs was further investigated using DLS, while the stability of colloidal particles as well as the surface charge on the particles, was measured by determining zeta-potential $(\zeta)$.

The results of DLS analysis are presented in Fig. 4, while measurements of $\zeta$ are displayed in Fig. 5. The obtained average hydrodynamic diameter of particles was the lowest for p-GQDs $(13.85 \mathrm{~nm})$, while for all gamma-irradiated GQDs the higher values are observed: $45.64 \mathrm{~nm}$, 44.21 and $71.65 \mathrm{~nm}$, for GQDs-25-EDA, GQDs-50-EDA and GQDs-200-

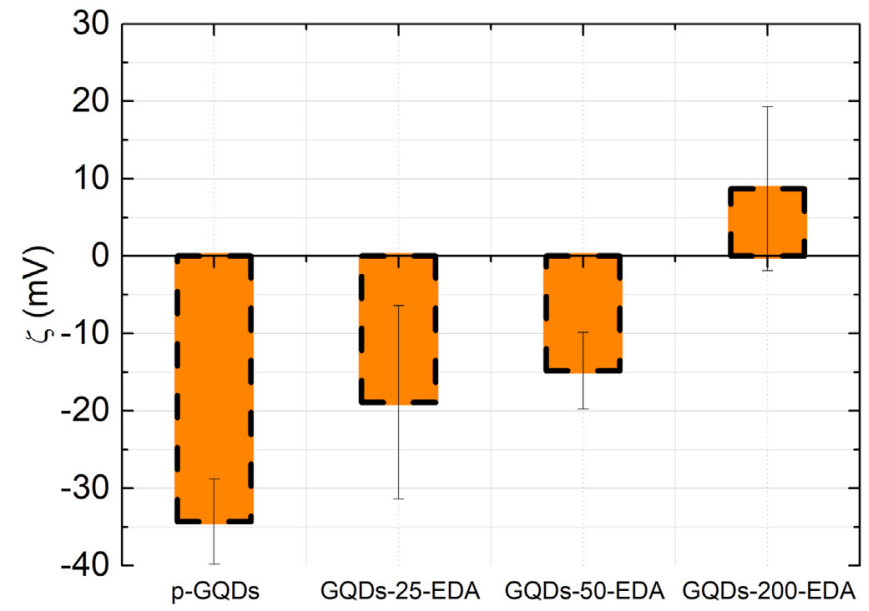

Fig. 5. Zeta potential values measured for p-GQDs, GQDs-25-EDA, GQDs-50EDA and GQDs-200-EDA.
EDA, respectively.

To estimate the surface charge density, $\zeta$ was measured, at $\mathrm{pH} 7$. Due to the presence of deprotonated oxygen functional groups such as carboxyl, GQDs are usually negatively charged $[63,64]$. The measured $\zeta$ for p-GQDs is $-34.6 \mathrm{mV}$. Gamma irradiation in the selected medium induced significant changes in $\zeta:-18.8,-15.1$ and $+9.1 \mathrm{mV}$ at the doses of 25, 50 and $200 \mathrm{kGy}$, respectively. While p-GQDs, GQDs-25EDA and GQDs-50-EDA are negatively charged, the dominant functional groups at the surface of GQD-200-EDA are positively charged moieties. Thus, it is plausible to say that gamma-irradiated GQDs possess a lower number of oxygen-containing functional groups than $\mathrm{p}$ GQDs. Additionally, GQDs-200-EDA are positively charged probably due to these events:

1. the lowering of negatively charged carboxyl groups;

2. the increasing of positively charged $\mathrm{N}$-functional groups in GQDs structure.

These changes will be investigated by FTIR and XPS in the following part of the manuscript.

\subsection{TEM measurement}

The morphology of gamma-irradiated GQDs is further investigated using TEM (Fig. 6). In the case of GQDs-25-EDA, particles with the lateral size between 23 and $44 \mathrm{~nm}$ are observed, while for GQDs-50EDA few layers dots with diameter from 28 to $40 \mathrm{~nm}$ are indicated in Fig. 6, b. As for GQDs-200-EDA, large disc-like structures are observed.

Based on AFM, DLS and TEM analyses, we concluded that gamma irradiation in all selected conditions leads to the increase in particle 

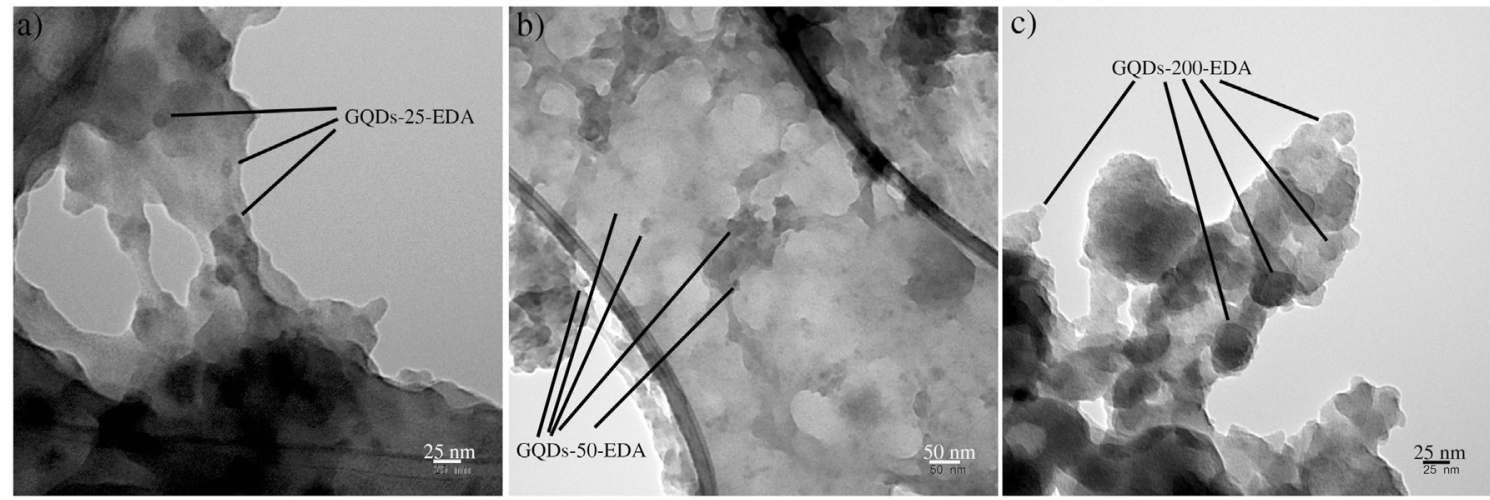

Fig. 6. TEM images of GQDs-25-EDA (a), GQDs-50-EDA (b) and GQDs-200-EDA (c).

size. At a lower dose, such as $25 \mathrm{kGy}$ a decrease in the heigh of particles was observed, while the lateral size was increased compared to p-GQDs (13.85 $\mathrm{nm}-45.64 \mathrm{~nm}$, based on DLS). The highest applied irradiation dose (200 kGy) induced a large increase in the height of GQDs (from $1.14 \mathrm{~nm}$ as observed for p-GQDs to $24 \mathrm{~nm}$, as observed for GQD-200EDA), as well as in the diameter. Considering the changes in surface charge of particles, the particles probably lost the electrostatic stabilization due to large changes in chemical structure.

\subsection{FTIR analysis}

The structure of GQDs was analyzed using FTIR spectroscopy (Fig. 7.). The bands at 3155,3283 and $3414 \mathrm{~cm}^{-1}$ detected in the spectrum of the p-GQDs stem from the vibrations of $\mathrm{O}-\mathrm{H}$ bonds in hydroxyl groups attached to carbon atoms of GQDs [65]. Next, we observed low-intensity bands located at 2970,2931 and $2860 \mathrm{~cm}^{-1}$. These bands are the result of vibrations in $\mathrm{C}-\mathrm{H}$ bonds in $-\mathrm{CH}$ and $-\mathrm{CH}_{2}$ groups [13,65]. Two bands, at 2697 and $2781 \mathrm{~cm}^{-1}$ were also observed and assigned to bonds in the aromatic aldehyde [13]. The band at $1700 \mathrm{~cm}^{-1}$ stems from the vibration of $\mathrm{C}=\mathrm{O}$ bonds in carboxyl functional groups [66]. At around $1570 \mathrm{~cm}^{-1}$ strong band is noticed and it stems from stretching vibration of the $\mathrm{C}=\mathrm{C}$ bonds in aromatic domains [66]. Finally, a band centered at $1010 \mathrm{~cm}^{-1}$ which associated with the vibration of $\mathrm{C}-\mathrm{O}$ bonds, and a second band located at $1365 \mathrm{~cm}^{-1}$, which is due to symmetric stretching of $\mathrm{O}-\mathrm{C}=\mathrm{O}$ groups were found [65].

In the FTIR spectra of GQDs gamma-irradiated with EDA, significant changes have been observed compared to the pristine material. Firstly, the bands at 3155,3283 and $3414 \mathrm{~cm}^{-1}$ completely vanished from all irradiated GQDs. The only low-intensity band, at around $3360 \mathrm{~cm}^{-1}$ is observed. This change can be interpreted as removing $\mathrm{OH}$ functional groups. A new band at $3360 \mathrm{~cm}^{-1}$ arises from N-H bonds [67]. All three low-intensity bands at 2970, 2931 and $2860 \mathrm{~cm}^{-1}$ are observed in the FTIR spectra of gamma-irradiated GQDs which is an indication that $\mathrm{CH}$ - bonds in GQDs are preserved after irradiation in the presented condition. The next difference is noticed for bands located at 2697 and $2781 \mathrm{~cm}^{-1}$ in the spectrum of p-GQDs. These bands have disappeared upon gamma irradiation of GQDs with EDA. Considering that those bands are assigned to aromatic aldehyde, we suggest that in the presented condition, $\mathrm{C}=\mathrm{O}$ bonds are removed due to reductive conditions. Next, at $1700 \mathrm{~cm}^{-1}$ the band is found only in the spectrum of GQDs200-EDA, for GQDs-25-EDA the band at $1630 \mathrm{~cm}^{-1}$ is noticed while in the spectrum of GQDs-50-EDA we do not observe any feature in this region. All three samples show the band at $1570 \mathrm{~cm}^{-1}$ which proves that $\pi$-conjugated domains are preserved after gamma irradiation. The band at 1365 is shifted at $1375 \mathrm{~cm}^{-1}$ and it has a lower intensity,

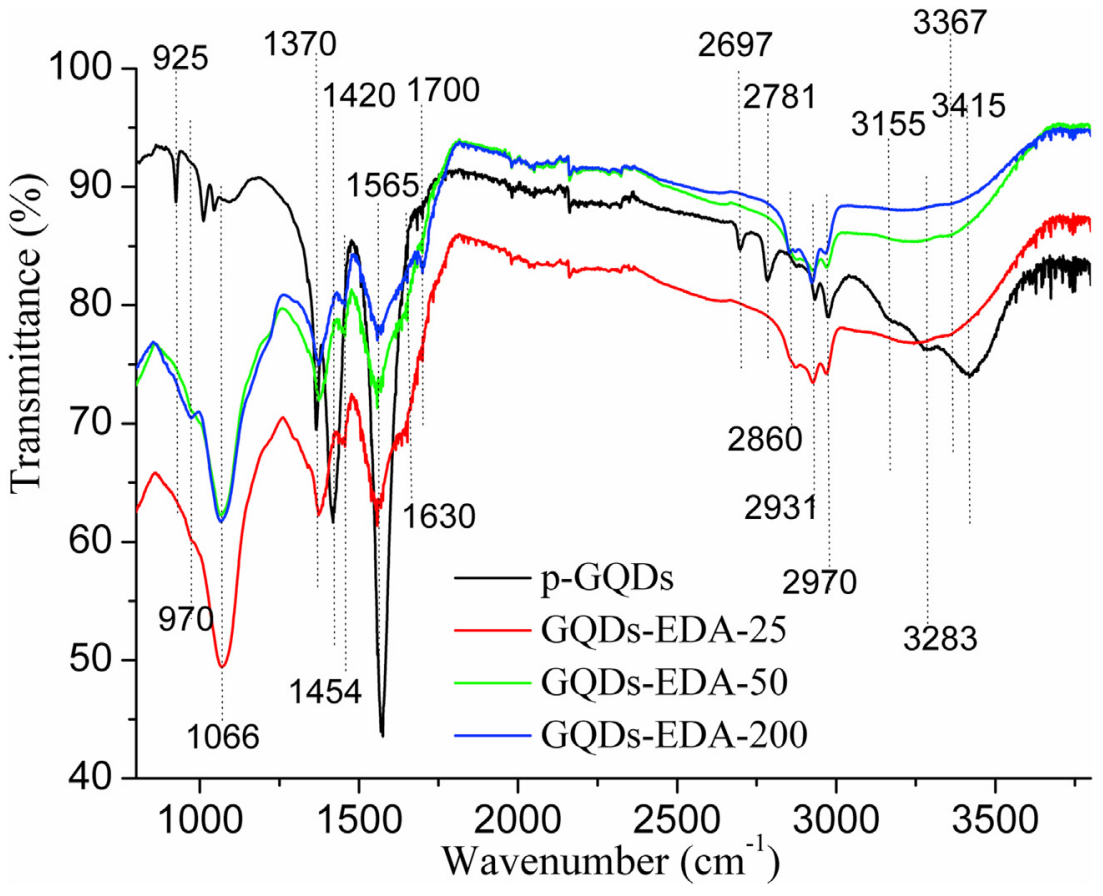

Fig. 7. FTIR spectra of p-GQDs (black curve), GQDs-25-EDA (red), GQDs-50-EDA (green) and GQDs-200-EDA (blue). 

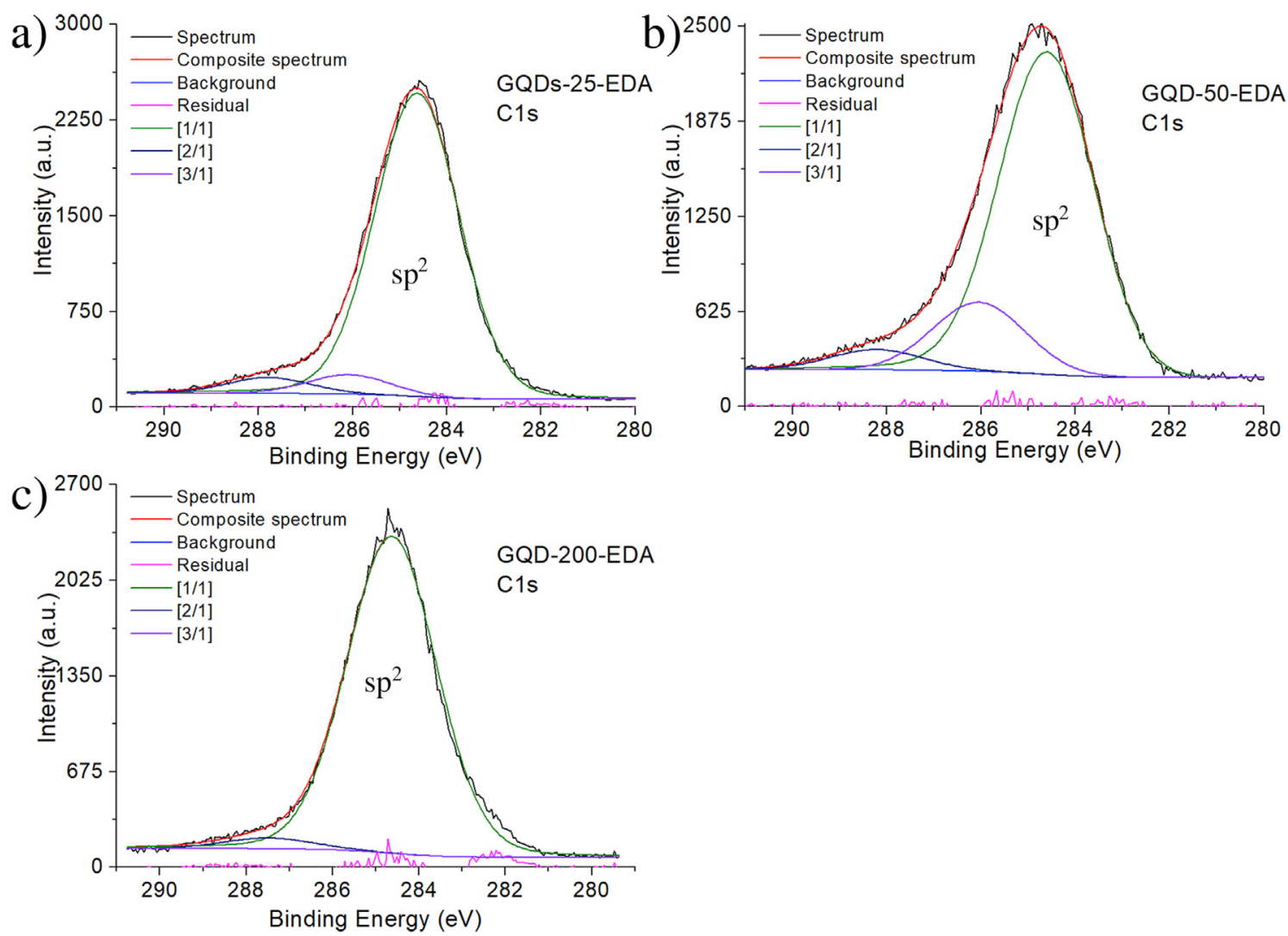

Fig. 8. High-resolution XPS spectra of GQDs-25-EDA (a), GQDs-50-EDA (b), and GQDs-200-EDA (c).

suggesting a reduction of $\mathrm{O}-\mathrm{C}=\mathrm{O}$ groups. The high-intensity band at $1066 \mathrm{~cm}^{-1}$ is present in all three spectra of gamma-irradiated GQDs. This band stems from the $\mathrm{C}-\mathrm{N}$ vibration of amine functional groups $[42,68]$. These results proved the successful incorporation of nitrogen atoms into the GQD structure by the presented irradiation process. Also, these spectra prove a significant lowering in O-containing functional groups.

\subsection{XPS analysis}

In Fig. 8(a-c), the high-resolution XPS spectra are shown. The analysis of the C1s band of GQDs-25-EDA showed the following composition: $\mathrm{C}=\mathrm{C}=89.42 \%$; $\mathrm{C}-\mathrm{O} / \mathrm{C}-\mathrm{N}=6.50 \%$; $\mathrm{C}=\mathrm{O}=4.45 \%$. In the sample of GQDs-50-EDA: $\mathrm{C}=\mathrm{C}=78.99 \%$; $\mathrm{C}-\mathrm{O} / \mathrm{C}-\mathrm{N}=16.26 \%$; $\mathrm{C}=\mathrm{O}$ $=4.75 \%$. In the case of GQDs-200-EDA: $\mathrm{C}=\mathrm{C}=96.91 \%$; $\mathrm{C}=\mathrm{O}=$ $3.09 \%$.

Based on XPS analysis, the chemical compostion of each GQD sample was analyzed and presented in Table 3. These results show that gamma irradiation in the presence of EDA and IPA induces the binding of amino groups in GQD structure while other oxidized $\mathrm{N}$-functional groups are not detected. Presented XPS data are in agreement with FTIR analysis, which also proves the presence of amino groups in gammairradiated samples. Also, in FTIR spectra, band at $1700 \mathrm{~cm}^{-1}$ is observed in $\mathrm{p}-\mathrm{GQD}$ and assigned to carbonyl groups of $\mathrm{COOH}$ functional

Table 3

The chemical composition of GQD samples in the atomic percentage.

\begin{tabular}{llll}
\hline Sample & $\% \mathrm{C}$ & $\% \mathrm{O}$ & $\% \mathrm{~N}$ \\
\hline p-GQDs [69] & 63.00 & 37.00 & 0.00 \\
GQDs-25-EDA & 84.42 & 12.57 & 3.01 \\
GQDs-50-EDA & 81.90 & 15.08 & 3.02 \\
GQDs-200-EDA & 85.21 & 11.63 & 3.16 \\
\hline
\end{tabular}

groups. In the case of GQDs-50-EDA the band in this area did not observe, but in the XPS spectrum $\mathrm{C}=\mathrm{O}$ groups are detected. Also, zeta potential is higher than in the case of GQDs-25-EDA, $-15.1 \mathrm{mV}$. Due to the negative value of particles charge, it is obvious that GQDs-50-EDA are still posses negatively charged carboxyl functional groups. Thus, the band assigned to $\mathrm{C}=\mathrm{O}$ vibrations in $\mathrm{COOH}$ groups is probably covered with band higher intensity, such as strong band at $1565 \mathrm{~cm}^{-1}$.

At $200 \mathrm{kGy}$, the GQDs structure is highly reduced, $\mathrm{sp}^{2}$ domains are restored and the band at $1700 \mathrm{~cm}^{-1}$ stems from the vibrations of the carbonyl, $\mathrm{C}=\mathrm{O}$ bonds which are in conjugation with aromatic graphene domains.

Additionally, a significant lowering in $\mathrm{O}$ content (11.63\%) is observed, as well as, an increase in $\%$ of $\mathrm{C}=\mathrm{C}$ bonds for GQDs irradiated at a dose of $200 \mathrm{kGy}$. This result suggests that the highest chemical reduction has occurred under these irradiation conditions.

Based on the presented results, we conclude that the highest amount of $\mathrm{O}$ functional groups such as $\mathrm{COOH}$ is in the sample of p-GQDs. At $\mathrm{pH}$ 7 , carboxyl groups are deprotonated and negatively charged, thus $\zeta$ is very low $(-34.6 \mathrm{mV})$. After the irradiation at a dose of $25 \mathrm{kGy}$, a significant lowering in $\mathrm{O}$ content is observed leading to GQDs with a lower number of $\mathrm{COOH}$ groups as well as a higher amount of positively charged amino groups. Both structural changes resulted in a higher value of $\zeta$. The treatment of GQDs with the dose of $50 \mathrm{kGy}$, the amount of $\mathrm{COOH}$ is further lowered and the number of amino groups is increased. The largest $\zeta$ is measured for GQDs-200-EDA $(+9.1 \mathrm{mV})$ and it is assigned to the largest lowering in negatively charged carboxyl groups and increased in positive ammonium groups.

\subsection{Antioxidative activity of GQDs modified by EDA and gamma irradiation}

The ability of modified GQDs to scavenge radical we conducted DPPH assay and the results of these measurements are presented in 

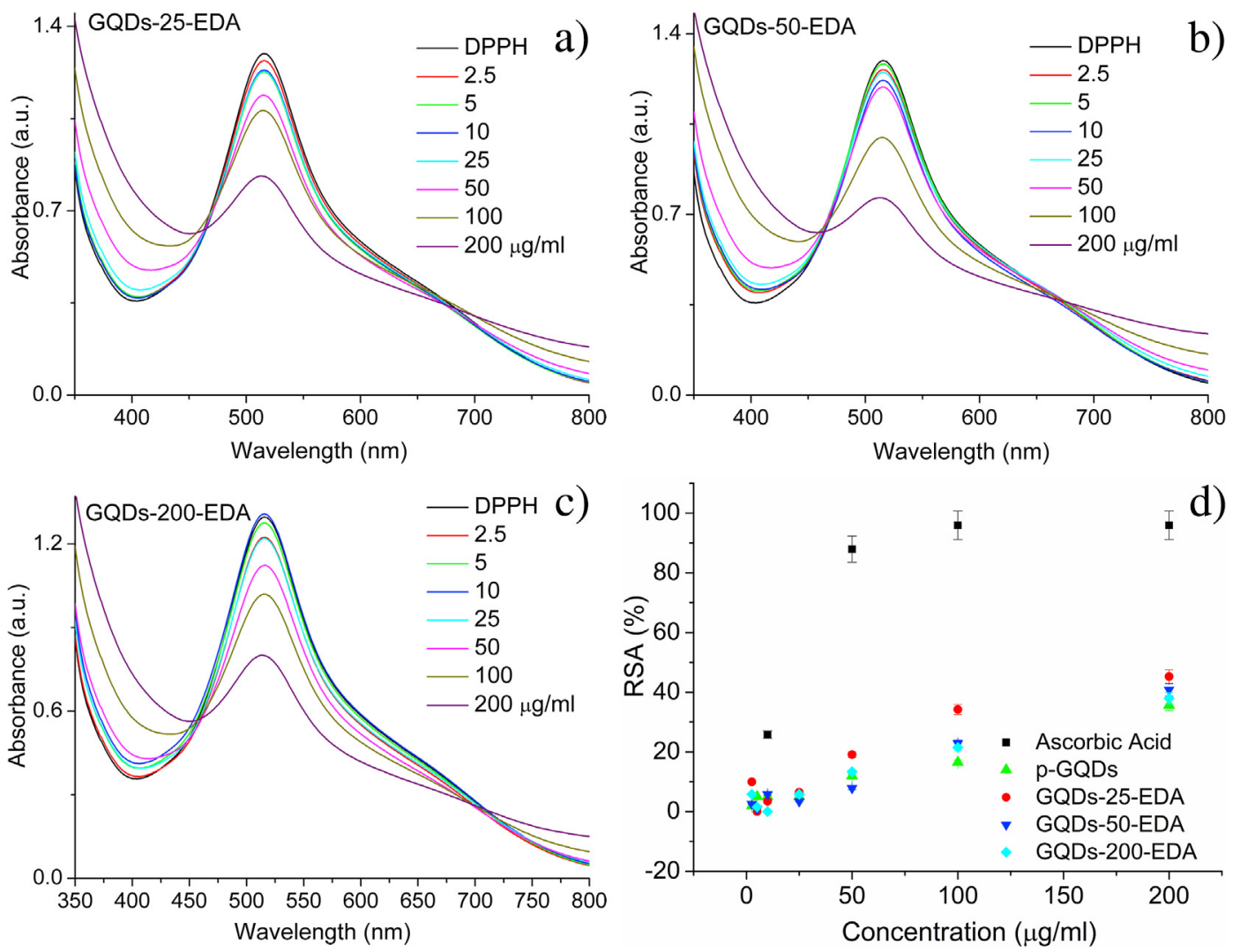

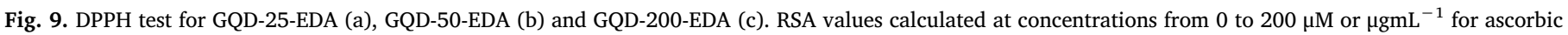
acid and GQD samples (d).

Fig. 9. This experiment is based on the measurement of the absorption spectra of DPPH radicals. A stabile DPPH radical shows a strong absorption band centered at $515 \mathrm{~nm}$. If the compound or material is able to quench free radicals, the intensity of absorption at $515 \mathrm{~nm}$ decreases. Herein, we mixed different amounts of GQDs with DPPH methanol solution and kept in dark for $1 \mathrm{~h}$. Afterward, the absorption spectra are recorded. A well-known antioxidant 1-ascorbic acid is used as a reference.

The intensity of the main DPPH band decreases with the concentration of gamma-irradiated GQDs, for all GQDs samples. This trend proves that modified GQDs possess antioxidative activity, similar to $\mathrm{p}$ GQDs [12]. By calculating RSA activity (see Methods section), as shown in Fig. 9d, we observed that all samples of GQDs have similar antioxidative activity. The increased antioxidative activity is observed when GQDs are chemically reduced and primary amino groups are attached [49]. This behavior is assigned to a strong hydrogen donation ability. Herein, proton donating ability of GQDs is not changed significantly compared to p-GQDs, but all dots are able to induce free radicals quenching.

\subsection{Detection of $\mathrm{Cu}(\mathrm{II})$ ions}

Fig. 10a and b show fluorescence emission spectra of mixture pGQDs and GQDs-200-EDA with different concentrations $\left(0-80.5 \mu \mathrm{mol} \mathrm{L} \mathrm{L}^{-1}\right)$ of $\mathrm{Cu}(\mathrm{II})$ ions under excitation wavelength at $360 \mathrm{~nm}$. The results show that the fluorescence intensity of emission decreases with the increase in $\mathrm{Cu}(\mathrm{II})$ ions concentration in both measured solutions, p-GQDs and GQDs-200-EDA. The relationship of integrated fluorescence intensity for p-GQDs and GQDs-200-EDA in the presence of $\mathrm{Cu}(\mathrm{II})$ ions is presented in Fig. $10 \mathrm{c}$ and $\mathrm{d}$, while the insets show linear plots of the $\mathrm{Cu}(\mathrm{II})$-induced fluorescence emission quenching obtained according to the Stern-Volmer equation [70]:
$\mathrm{A} / \mathrm{A}_{0}=1+\mathrm{K}_{\mathrm{sv}}[\mathrm{Cu}(\mathrm{II})]$

where $\mathrm{A}_{0}$ and $\mathrm{A}$ correspond to the integrated fluorescence intensities in the absence $\left(\mathrm{A}_{0}\right)$ and in the presence of the $\mathrm{Cu}(\mathrm{II})$ ions (A) with a different region of concentrations [Cu(II)], while $\mathrm{K}_{\mathrm{sv}}$ is the Stern-Volmer quenching constant, corresponding to the slope (S) from linear data fitting using Eq. (2).

The collected data follow the linear model of Stern-Volmer equation in the $0.3-6.4 \mu \mathrm{mol} \mathrm{L}^{-1}$ and $0.3-16.1 \mu \mathrm{mol} \mathrm{L}^{-1}$ ranges for p-GQDs and GQDs-200-EDA, respectively.

The limit of detection (LOD) was calculated using the following equation:

$\mathrm{LOD}=3 \mathrm{~S}_{\mathrm{D}} / \mathrm{K}_{\mathrm{sv}}$

where $S_{D}$ represents the standard deviation. The obtained LOD is evaluated to be $0.187 \mathrm{nmol} \mathrm{L}^{-1}\left(\mathrm{R}^{2}=0.974\right)$ and $153 \mathrm{nmol} \mathrm{L}{ }^{-1}\left(\mathrm{R}^{2}=0.984\right)$ for $\mathrm{p}$-GQDs and GQDs-200-EDA, respectively. The LOD values are in a similar range with recently published results [71,72]. A near 1 determination coefficient $\left(R^{2}\right)$ qualifies $p$ GQDs and GQDs-200-EDA nanoparticles as efficient quenchers.

\section{Discussion and conclusions}

By gamma irradiation of GQDs in the presence of EDA, IPA, and Ar atmosphere, the chemical modification of dots structure is achieved; primary amines are attached to the GDQs scaffold. Although different doses of irradiation were applied, similar amounts of $\mathrm{N}$-atoms are detected in all samples (3.01-3.16 at\%). Thus, gamma irradiation enables the GQD functionalization with amino groups in the presence of EDA, while the applied dose does not affect the number of incorporated amino groups. This result is different from the results obtained by gamma irradiation of GO with EDA, where the number of N-groups depended on the applied doses [41]. Thus, we assume that the amount 

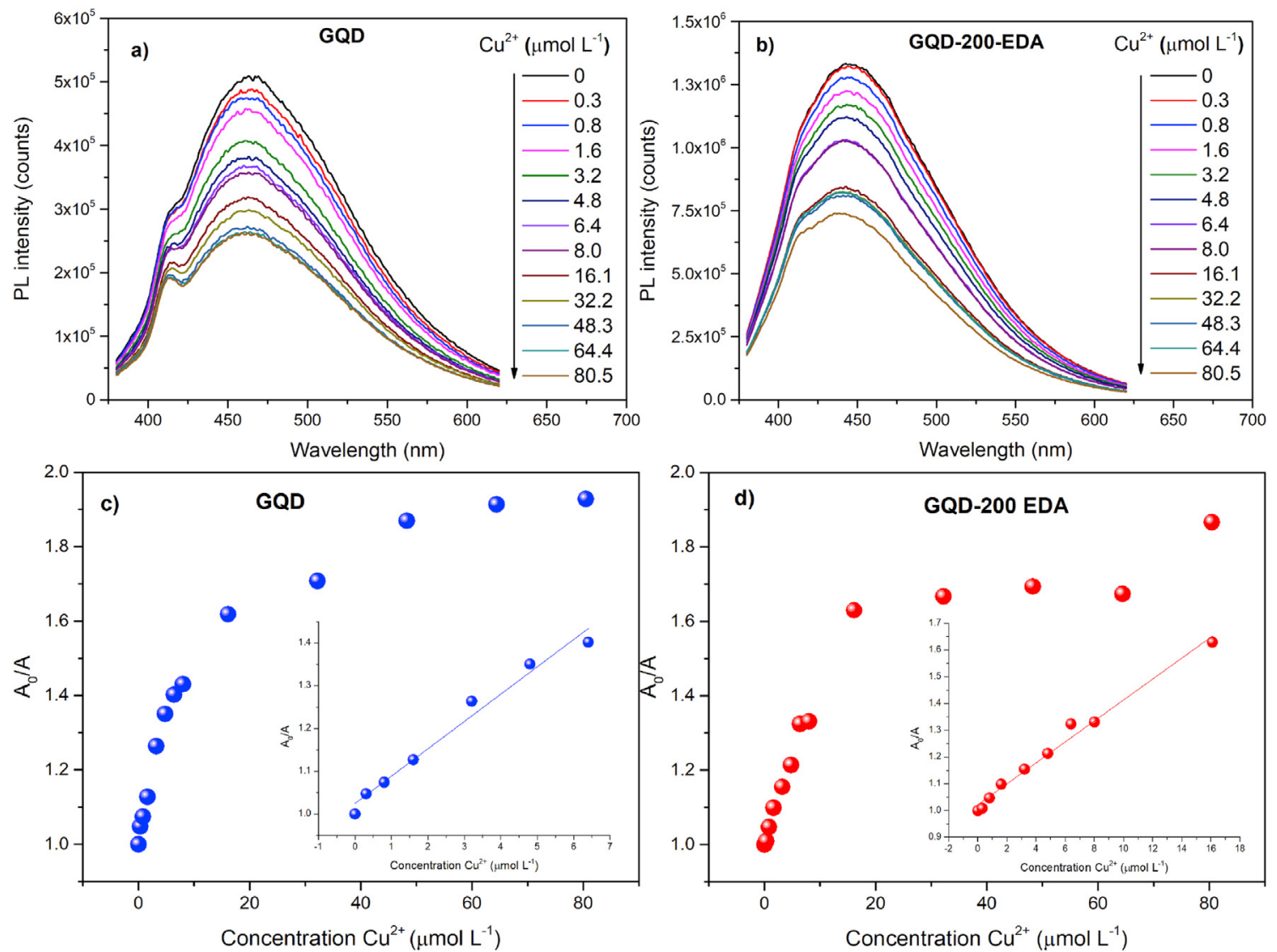

Fig. 10. The fluorescence emission spectra of the: a) p-GQDs and b) GQDs-200-EDA in the presence of various $\mathrm{Cu}(\mathrm{II})$ ions concentrations ( $0-80.5 \mu$ molL ${ }^{-1}$ ). The dependence of integrated fluorescence intensity for c) p-GQDs and d) GQDs-200-EDA in the presence of various concentrations of $\mathrm{Cu}(\mathrm{II})$ ions $\left(0-80.5 \mu \mathrm{molL}{ }^{-1}\right)$ and corresponding insets show Stern-Volmer plots of the Cu(II)-induced fluorescence emission quenching. Error bars are too small to be shown on plots.

of EDA added before irradiation may have an impact on the number of incorporated $\mathrm{N}$-atoms. Also, the structure of GQDs is additionally modulated due to reduction irradiation condition and relative abundance of $\mathrm{sp}^{2} \mathrm{C}$-atoms is significantly increased compared to $\mathrm{p}$-GQDs, from $63 \%$ (obtained for p-GQDs) to $79 \%$ or higher. These results prove that significant restoring of $\mathrm{sp}^{2} \mathrm{C}$-atoms is achieved in selected conditions. Also, at a dose of $200 \mathrm{kGy}$, the percentage of $\mathrm{sp}^{2} \mathrm{C}$-atoms is the highest (96\%) while C-O bonds are not detected. In this case, a dose of gamma irradiation significantly affected the amount of both $\mathrm{sp}^{2} \mathrm{C}$ - and $\mathrm{C}$ in O-functional groups.

Due to lowering in the abundance of $\mathrm{O}$ - functional groups, gammairradiated GQDs are less dispersible in water, where AFM analysis reveals the presence of agglomerated nanoparticles, while DLS shows a large increase in hydrodynamic volume (from $13.85 \mathrm{~nm}$ measured for p-GQDs to $71.65 \mathrm{~nm}$, for GQDs-200-EDA). This may affect the ability of GQDs to penetrate the cells as well as reduced the quality of the thin films when these dispersions are used for deposition. The large changes in surface charge are also noticed by investigation of $\zeta$, which shows that charge changed from $-34.6 \mathrm{mV}$ for $\mathrm{p}$-GQDs to $-18.8,-15.1$ and $+9.1 \mathrm{mV}$ for GQDs-25-EDA, GQDs-50-EDA and GQDs-200-EDA, respectively. In the case of p-GQDs, the negative charge of particles is due to deprotonated carboxyl groups, while in the case of gamma-irradiated GQDs, the higher values are a consequence of lowering in the number of carboxyl functional groups, as well as the increase in the number of positively charged primary amine groups. The highest value of surface charge for GQDs-200-EDA nanoparticles is related to the largest lowering in carboxyl group content, and the attachment of amine groups.

One of the most significant changes is noticed in the QY of PL. We observed the increase in both intensity and QY of PL. The highest QY was measured for GQDs-200-EDA, 18.4\% at the excitation wavelength of $320 \mathrm{~nm}$. Our previous study showed that gamma irradiation with IPA also increased QY of PL, from 1.0 to $6.1 \%$, after irradiation at a dose of 200 kGy [13]. The changes in PL can be explained by increased $\mathrm{sp}^{2} \mathrm{C}$ atoms and the lowering of oxygen-containing functional groups $[13,59]$. Unexpectedly, the relative QY was the lowest for GQDs-50EDA possible due to defect formation in graphene lattice and consequently the lowering in $\mathrm{sp}^{2}$ hybridized $\mathrm{C}$ atoms. It was observed that gamma-irradiated GQDs possess the ability to quench radical species as well as to detect $\mathrm{Cu}(\mathrm{II})$ ions.

In conclusion, for the first time gamma irradiation was successfully used in order to introduce N-functional groups in GQDs structure. Apart from amine groups, irradiation caused a significant chemical reduction of dots. These chemical changes were yield to 9-fold increased photoluminescence QY and the formation of positively charged nanoparticles with the ability to quench free radicals and detect $\mathrm{Cu}$ (II) ions. Owing to the highest increase in the PL intensity and the ability to quench PL in the presence of metal ions, GQDs-200-EDA is the most effective material among our library of modified GDQs to be applied for the detection of environmentally relevant pollutants.

\section{Declaration of competing interest}

The authors declare that they have no known competing financial interests or personal relationships that could have appeared to influence the work reported in this paper.

\section{Acknowledgments}

This work was financially supported by the Ministry of Education, 
Science and Technological Development of the Republic of Serbia (Grant No. 451-03-68/2020-14/200017 and 451-03-68/2020-14/ 200026). The Italian Ministry of University and Research (MURST, exMIUR) is acknowledged by A.B. for funding his research activities (PON "AIM: Attrazione e Mobilità Internazionale", call AIM1809078-2, CUP B78D19000280001). ATeN Center (University of Palermo; project "Mediterranean Center for Human Health Advanced Biotechnologies (CHAB)" PON R\&C 2007-2013) is acknowledged for hospitality and service.

Authors thanks to prof. Tatjana Verbić from the Faculty of Chemistry, the University of Belgrade for her support during the PL measurements.

\section{References}

[1] L.A. Ponomarenko, F. Schedin, M.I. Katsnelson, R. Yang, E.W. Hill, K.S. Novoselov, A.K. Geim, Chaotic Dirac billiard in graphene quantum dots, Science 320 (2008) 356-358, https://doi.org/10.1126/science.1154663.

[2] Y. Yan, J. Gong, J. Chen, Z. Zeng, W. Huang, K. Pu, J. Liu, P. Chen, Recent advances on graphene quantum dots: from chemistry and physics to applications, Adv. Mater. 31 (2019) 1808283, https://doi.org/10.1002/adma.201808283.

[3] X. Zhou, Y. Zhang, C. Wang, X. Wu, Y. Yang, B. Zheng, H. Wu, S. Guo, J. Zhang, Photo-Fenton reaction of graphene oxide: a new strategy to prepare graphene quantum dots for DNA cleavage, ACS Nano 6 (2012) 6592-6599, https://doi.org/ $10.1021 / \mathrm{nn} 301629 \mathrm{v}$.

[4] P. Elvati, E. Baumeister, A. Violi, Graphene quantum dots: effect of size, composition and curvature on their assembly, RSC Adv. 7 (2017) 17704-17710, https://doi. org/10.1039/C7RA01029J.

[5] S.P. Jovanović, O. Hanssler, M.D. Budimir, D. Kleut, J. Prekodravac, B.M. Todorović Marković, Reduction of Graphene Oxide and Graphene Quantum Dots Using Nascent Hydrogen: Investigation of Morphological and Structural Changes 14th Multinational Congress on MicroscopyBelgrade, Serbia, 2019.

[6] L. Li, G. Wu, G. Yang, J. Peng, J. Zhao, J.-J. Zhu, Focusing on luminescent graphene quantum dots: current status and future perspectives, Nanoscale 5 (2013) 4015-4039, https://doi.org/10.1039/c3nr33849e.

[7] S. Maleki Dizaj, A. Mennati, S. Jafari, K. Khezri, K. Adibkia, Antimicrobial activity of carbon-based nanoparticles, Adv. Pharmaceut. Bull. 5 (2015) 19-23, https://doi. org/10.5681/apb.2015.003.

[8] S. Iravani, R.S. Varma, Green synthesis, biomedical and biotechnological applications of carbon and graphene quantum dots. A review, Environ. Chem. Lett. (2020) 1-25, https://doi.org/10.1007/s10311-020-00984-0.

[9] S. Shen, J. Wang, Z. Wu, Z. Du, Z. Tang, J. Yang, Graphene quantum dots with high yield and high quality synthesized from low cost precursor of aphanitic graphite, Nanomaterials 10 (2020) 375, https://doi.org/10.3390/nano10020375.

[10] R. Ye, C. Xiang, J. Lin, Z. Peng, K. Huang, Z. Yan, N.P. Cook, E.L.G. Samuel, C.C. Hwang, G. Ruan, G. Ceriotti, A.-R.O. Raji, A.A. Martí, J.M. Tour, Coal as an abundant source of graphene quantum dots, Nat. Commun. 4 (2013) 2943, https:// doi.org/10.1038/ncomms3943.

[11] S.P. Jovanović, Photoluminescence of carbon-based nanomaterials: fullerenes, carbon nanotubes, graphene, graphene oxide, graphene and carbon quantum dots, in: E. Marsden (Ed.), Photoluminescence: Advances in Research and Applications, Nova Science Publishers, Inc., New York, USA, 2018, pp. 167-196.

[12] S.P. Jovanović, Z. Syrgiannis, M.D. Budimir, D.D. Milivojević, D.J. Jovanović, V.B. Pavlović, J.M. Papan, M. Bartenwerfer, M.M. Mojsin, M.J. Stevanović, B.M. Todorović Marković, Graphene quantum dots as singlet oxygen producer or radical quencher - the matter of functionalization with urea/thiourea, Mater. Sci. Eng. C 109 (2020) 110539, https://doi.org/10.1016/j.msec.2019.110539.

[13] S.P. Jovanović, Z. Syrgiannis, Z.M. Marković, A. Bonasera, D.P. Kepic, M.D. Budimir, D.D. Milivojević, V.D. Spasojević, M.D. Dramićanin, V.B. Pavlović, B.M. Todorović Marković, Modification of structural and luminescence properties of graphene quantum dots by gamma irradiation and their application in a photodynamic therapy, ACS Appl. Mater. Interfaces 7 (2015) 25865-25874, https://doi. org/10.1021/acsami.5b08226.

[14] M. Li, T. Chen, J.J. Gooding, J. Liu, Review of carbon and graphene quantum dots for sensing, ACS Sens. 4 (2019) 1732-1748, https://doi.org/10.1021/acssensors. 9 b00514.

[15] H. Sun, L. Wu, W. Wei, X. Qu, Recent advances in graphene quantum dots for sensing, Mater. Today 16 (2013) 433-442, https://doi.org/10.1016/j.mattod.2013. 10.020 .

[16] N.A.A. Anas, Y.W. Fen, N.A.S. Omar, W.M.E.M.M. Daniyal, N.S.M. Ramdzan, S. Saleviter, Development of graphene quantum dots-based optical sensor for toxic metal ion detection, Sensors 19 (2019) 3850, https://doi.org/10.3390/s19183850.

[17] H. Sun, L. Wu, W. Wei, X. Qu, Recent advances in graphene quantum dots for sensing, Mater. Today 16 (2013) 433-442, https://doi.org/10.1016/j.mattod.2013. 10.020 .

[18] H. Shi, G. Zhao, M. Liu, L. Fan, T. Cao, Aptamer-based colorimetric sensing of acetamiprid in soil samples: sensitivity, selectivity and mechanism, J. Hazard Mater. 260 (2013) 754-761, https://doi.org/10.1016/j.jhazmat.2013.06.031.

[19] Y. Kim, R.C. Johnson, J.T. Hupp, Gold nanoparticle-based sensing of "Spectroscopically silent" heavy metal ions, Nano Lett. 1 (2001) 165-167, https:// doi.org/10.1021/nl0100116.
[20] Z. Zhang, Z. Chen, S. Wang, C. Qu, L. Chen, On-site visual detection of hydrogen sulfide in air based on enhancing the stability of gold nanoparticles, ACS Appl. Mater. Interfaces 6 (2014) 6300-6307, https://doi.org/10.1021/am500564w.

[21] F. Tian, J. Zhou, B. Jiao, Y. He, A nanozyme-based cascade colorimetric aptasensor for amplified detection of ochratoxin A, Nanoscale 11 (2019) 9547-9555, https:// doi.org/10.1039/C9NR02872B.

[22] A. Pacquit, J. Frisby, D. Diamond, K.T. Lau, A. Farrell, B. Quilty, D. Diamond, Development of a smart packaging for the monitoring of fish spoilage, Food Chem. 102 (2007) 466-470, https://doi.org/10.1016/j.foodchem.2006.05.052.

[23] I.A. Bulushi, S. Poole, H.C. Deeth, G.A. Dykes, Biogenic amines in fish: roles in intoxication, spoilage, and nitrosamine formation-a review, Crit. Rev. Food Sci. Nutr. 49 (2009) 369-377, https://doi.org/10.1080/10408390802067514.

[24] N.T. Greene, K.D. Shimizu, Colorimetric molecularly imprinted polymer sensor array using dye displacement, J. Am. Chem. Soc. 127 (2005) 5695-5700, https:// doi.org/10.1021/ja0468022.

[25] N.A. Rakow, A. Sen, M.C. Janzen, J.B. Ponder, K.S. Suslick, Molecular recognition and discrimination of amines with a colorimetric array, Angew Chem. Int. Ed. Engl. 44 (2005) 4528-4532, https://doi.org/10.1002/anie.200500939.

[26] V. Valderrey, A. Bonasera, S. Fredrich, S. Hecht, Light-activated sensitive probes for amine detection, Angew Chem. Int. Ed. Engl. 56 (2017) 1914-1918, https://doi. org/10.1002/anie.201609989.

[27] Y. Che, X. Yang, S. Loser, L. Zang, Expedient vapor probing of organic amines using fluorescent nanofibers fabricated from an n-type organic semiconductor, Nano Lett. 8 (2008) 2219-2223, https://doi.org/10.1021/nl080761g.

[28] S. Fredrich, A. Bonasera, V. Valderrey, S. Hecht, Sensitive assays by nucleophileinduced rearrangement of photoactivated diarylethenes, J. Am. Chem. Soc. 140 (2018) 6432-6440, https://doi.org/10.1021/jacs.8b02982.

[29] H. Sun, N. Gao, L. Wu, J. Ren, W. Wei, X. Qu, Highly photoluminescent aminofunctionalized graphene quantum dots used for sensing copper ions, Chem. Eur J. 19 (2013) 13362-13368, https://doi.org/10.1002/chem.201302268.

[30] L. Zhou, J. Geng, B. Liu, Graphene quantum dots from polycyclic aromatic hydrocarbon for bioimaging and sensing of $\mathrm{Fe} 3+$ and hydrogen peroxide, Part. Part. Syst. Char. 30 (2013) 1086-1092, https://doi.org/10.1002/ppsc.201300170.

[31] M. Roushani, S. Kohzadi, S. Haghjoo, A. Azadbakht, Dual detection of malation and $\mathrm{Hg}$ (II) by fluorescence switching of graphene quantum dots, Environ. Nanotechnol. Monit. Manag. 10 (2018) 308-313, https://doi.org/10.1016/j.enmm.2018.08.002.

[32] K. Wang, J. Dong, L. Sun, H. Chen, Y. Wang, C. Wang, L. Dong, Effects of elemental doping on the photoluminescence properties of graphene quantum dots, RSC Adv. 6 (2016) 91225-91232, https://doi.org/10.1039/C6RA19673J.

[33] X. Xu, F. Gao, X. Bai, F. Liu, W. Kong, M. Li, Tuning the photoluminescence of graphene quantum dots by photochemical doping with nitrogen, Materials 10 (2017) 1328, https://doi.org/10.3390/ma10111328.

[34] R.L. Calabro, D.-S. Yang, D.Y. Kim, Controlled nitrogen doping of graphene quantum dots through laser ablation in aqueous solutions for photoluminescence and electrocatalytic applications, ACS Appl. Nano Mater. 2 (2019) 6948-6959, https://doi.org/10.1021/acsanm.9b01433.

[35] Y. Luo, Y. Xu, M. Li, L. Sun, G. Hu, T. Tang, J. Wen, X. Li, Tuning the photoluminescence of graphene quantum dots by fluorination, J. Nanomater. 2017 (2017) 1-6, https://doi.org/10.1155/2017/9682846.

[36] A. Ananthanarayanan, Y. Wang, P. Routh, M.A. Sk, A. Than, M. Lin, J. Zhang, J. Chen, H.D. Sun, P. Chen, Nitrogen and phosphorus co-doped graphene quantum dots: synthesis from adenosine triphosphate, optical properties, and cellular imaging, Nanoscale 7 (2015) 8159-8165, https://doi.org/10.1039/c5nr01519g.

[37] Y.M. Zhang, J.H. Zhao, H.L. Sun, Z.Q. Zhu, J. Zhang, Q.J. Liu, B, N, S, Cl doped graphene quantum dots and their effects on gas-sensing properties of Ag-LaFeO3, Sensor. Actuator. B Chem. 266 (2018) 364-374, https://doi.org/10.1016/j.snb. 2018.03.109.

[38] S. Kundu, R.M. Yadav, T.N. Narayanan, M.V. Shelke, R. Vajtai, P.M. Ajayan, V.K. Pillai, Synthesis of N, F and S co-doped graphene quantum dots, Nanoscale 7 (2015) 11515-11519, https://doi.org/10.1039/c5nr02427g.

[39] J. Prekodravac, B. Vasiljević, Z. Marković, D. Jovanović, D. Kleut, Z. Špitalský, M. Mičušik, M. Danko, D. Bajuk-Bogdanović, B. Todorović-Marković, Green and facile microwave assisted synthesis of (metal-free) N-doped carbon quantum dots for catalytic applications, Ceram. Int. 45 (2019) 17006-17013, https://doi.org/10. 1016/j.ceramint.2019.05.250.

[40] C. Wu, R. Chen, C. Ma, R. Cheng, X. Gao, T. Wang, Y. Liu, P. Huo, Y. Yan, Construction of upconversion nitrogen doped graphene quantum dots modified BiVO4 photocatalyst with enhanced visible-light photocatalytic activity, Ceram. Int. 45 (2019) 2088-2096, https://doi.org/10.1016/j.ceramint.2018.10.112.

[41] M.A. Sk, A. Ananthanarayanan, L. Huang, K.H. Lim, P. Chen, Revealing the tunable photoluminescence properties of graphene quantum dots, J. Mater. Chem. C 2 (2014) 6954-6960, https://doi.org/10.1039/c4tc01191k.

[42] N. Ahmad Daud, B.W. Chieng, N.A. Ibrahim, Z.A. Talib, E.N. Muhamad, Z.Z. Abidin, Functionalizing graphene oxide with alkylamine by gamma-ray irradiation method, Nanomaterials 7 (2017) 135, https://doi.org/10.3390/nano7060135.

[43] S. Jovanović, Z. Marković, M.D. Budimir, Z. Spitalsky, B. Vidoeski, B.M. Todorović Marković, Effects of low gamma irradiation dose on the photoluminescence properties of graphene quantum dots, Opt. Quant. Electron. 48 (2016) 259, https://doi. org/10.1007/s11082-016-0516-z.

[44] K. Mishra, H. Ojha, N.K. Chaudhury, Estimation of antiradical properties of antioxidants using DPPH assay: a critical review and results, Food Chem. 130 (2012) 1036-1043, https://doi.org/10.1016/j.foodchem.2011.07.127.

[45] H.T. Li, X.D. He, Z.H. Kang, H. Huang, Y. Liu, J.L. Liu, S.Y. Lian, C.H.A. Tsang, X.B. Yang, S.T. Lee, Water-soluble fluorescent carbon quantum dots and photocatalyst design, Angew. Chem. Int. Ed. 49 (2010) 4430-4434, https://doi.org/10. 1002/anie.200906154. 
[46] A.T.R. Williams, S.A. Winfield, J.N. Miller, Relative fluorescence quantum yields using a computer-controlled luminescence spectrometer, Analyst 108 (1983) 1067-1071, https://doi.org/10.1039/AN9830801067.

[47] D. Magde, G.E. Rojas, P.G. Seybold, Solvent dependence of the fluorescence lifetimes of xanthene dyes, Photochem. Photobiol. 70 (1999) 737-744, https://doi. org/10.1111/j.1751-1097.1999.tb08277.x.

[48] http://www.gwyddion.net.

[49] V. Ruiz, L. Yate, I. García, G. Cabanero, H.-J. Grande, Tuning the antioxidant activity of graphene quantum dots: protective nanomaterials against dye decoloration, Carbon 116 (2017) 366-374, https://doi.org/10.1016/j.carbon.2017.01.090.

[50] Z. Gan, H. Xu, Y. Hao, Mechanism for excitation-dependent photoluminescence from graphene quantum dots and other graphene oxide derivates: consensus, debates and challenges, Nanoscale 8 (2016) 7794-7807, https://doi.org/10.1039/ C6NR00605A.

[51] S. Lai, Y. Jin, L. Shi, R. Zhou, Y. Zhou, D. An, Mechanisms behind excitation- and concentration-dependent multicolor photoluminescence in graphene quantum dots, Nanoscale 12 (2020) 591-601, https://doi.org/10.1039/C9NR08461D.

[52] L. Minati, A. Del Piano, Facile synthesis of water-soluble, highly-fluorescent graphene quantum dots from graphene oxide reduction for efficient cell labelling, Chimia 5 (2019) 77, https://doi.org/10.3390/c5040077.

[53] S. Zhu, Y. Song, J. Wang, H. Wan, Y. Zhang, Y. Ning, B. Yang, Photoluminescence mechanism in graphene quantum dots: quantum confinement effect and surface/ edge state, Nano Today 13 (2017) 10-14, https://doi.org/10.1016/j.nantod.2016. 12.006 .

[54] S. Wang, I. Cole, D. Zhao, Q. Li, The dual roles of functional groups in the photoluminescence of graphene quantum dots, Nanoscale 8 (2015) 7449-7458, https:// doi.org/10.1039/C5NR07042B.

[55] G. Rajender, P.K. Giri, Formation mechanism of graphene quantum dots and their edge state conversion probed by photoluminescence and Raman spectroscopy, J. Mater. Chem. C 4 (2016) 10852-10865, https://doi.org/10.1039/C6TC03469A.

[56] G. Yang, C. Wu, X. Luo, X. Liu, Y. Gao, P. Wu, C. Cai, S.S. Saavedra, Exploring the emissive states of heteroatom-doped graphene quantum dots, J. Phys. Chem. C 122 (2018) 6483-6492, https://doi.org/10.1021/acs.jpcc.8b01385.

[57] S. Yang, C. Zhu, G. Wang, R. Mo, P. He, J. Sun, Z. Di, N. Yuan, J. Ding, G. Ding, $\mathrm{X}$. Xie, Negative induction effect of graphite $\mathrm{N}$ on graphene quantum dots: tunable band gap photoluminescence, J. Mater. Chem. C 3 (2015) 8810-8816, https://doi. org/10.1039/C5TC01933H.

[58] K. Hola, A. Bourlinos, O. Kozák, K. Berka, K. Siskova, M. Havrdova, J. Tucek, K. Safarova, M. Otyepka, E. Giannelis, R. Zboril, Photoluminescence effects of graphitic core size and surface functional groups in carbon dots: $\mathrm{COO}-$ induced red-shift emission, Carbon 70 (2014) 279-286, https://doi.org/10.1016/j.carbon. 2014.01.008.

[59] V. Stengl, S. Bakardjieva, J. Henych, K. Lang, M. Kormunda, Blue and green luminescence of reduced graphene oxide quantum dots, Carbon 63 (2013) 537-546, https://doi.org/10.1016/j.carbon.2013.07.031.

[60] S. Zhu, J. Shao, Y. Song, X. Zhao, J. Du, L. Wang, H. Wang, K. Zhang, J. Zhang,
B. Yang, Investigating the surface state of graphene quantum dots, Nanoscale 7 (2015) 7927-7933, https://doi.org/10.1039/C5NR01178G.

[61] L. Wang, S.-J. Zhu, H.-Y. Wang, S.-N. Qu, Y.-L. Zhang, J.-H. Zhang, Q.-D. Chen, H.L. Xu, W. Han, B. Yang, H.-B. Sun, Common origin of green luminescence in carbon nanodots and graphene quantum dots, ACS Nano 8 (2014) 2541-2547, https://doi. org $/ 10.1021 / \mathrm{nn} 500368 \mathrm{~m}$.

[62] S. Yang, W. Li, C. Ye, G. Wang, H. Tian, C. Zhu, P. He, G. Ding, X. Xie, Y. Liu, Y. Lifshitz, S.-T. Lee, Z. Kang, M. Jiang, C3N-a 2D crystalline, hole-free, tunablenarrow-bandgap semiconductor with ferromagnetic properties, Adv. Mater. 29 (2017) 1605625, https://doi.org/10.1002/adma.201605625.

[63] W. Kong, Y. Wang, L. Wang, Y. Li, Y. Li, W. Xue, Investigation of photoluminescence behavior of reduced graphene quantum dots, Inorg. Chem. Commun. 99 (2019) 199-205, https://doi.org/10.1016/j.inoche.2018.10.019.

[64] D.K.L. Chan, J.C. Yu, Y. Li, Z. Hu, A metal-free composite photocatalyst of graphene quantum dots deposited on red phosphorus, J. Environ. Sci. (China) 60 (2017) 91-97, https://doi.org/10.1016/j.jes.2016.11.025.

[65] Y. Zhang, C. Yang, D. Yang, Z. Shao, Y. Hu, J. Chen, L. Yuwen, L. Weng, Z. Luo, L. Wang, Reduction of graphene oxide quantum dots to enhance the yield of reactive oxygen species for photodynamic therapy, Phys. Chem. Chem. Phys. 20 (2018) 17262-17267, https://doi.org/10.1039/c8cp01990h.

[66] V. Tucureanu, A. Matei, A.M. Avram, FTIR spectroscopy for carbon family study, Crit. Rev. Anal. Chem. 46 (2016) 502-520, https://doi.org/10.1080/10408347. 2016.1157013.

[67] Y.-P. Zhang, J.-M. Ma, Y.-S. Yang, J.-X. Ru, X.-Y. Liu, Y. Ma, H.-C. Guo, Synthesis of nitrogen-doped graphene quantum dots (N-GQDs) from marigold for detection of $\mathrm{Fe} 3+$ ion and bioimaging, Spectrochim. Acta A Mol. Biomol. Spectrosc. 217 (2019) 60-67, https://doi.org/10.1016/j.saa.2019.03.044.

[68] Z. Qian, J. Ma, X. Shan, L. Shao, J. Zhou, J. Chen, H. Feng, Surface functionalization of graphene quantum dots with small organic molecules from photoluminescence modulation to bioimaging applications: an experimental and theoretical investigation, RSC Adv. 3 (2013) 14571-14579, https://doi.org/10.1039/ c3ra42066c.

[69] S.P. Jovanović, Z.M. Marković, Z. Syrgiannis, M.D. Dramićanin, F. Arcudi, V. LaParola, M.D. Budimir, B.M. Todorović Marković, Enhancing photoluminescence of graphene quantum dots by thermal annealing of the graphite precursor, Mater. Res. Bull. 93 (2017) 183-193, https://doi.org/10.1016/j. materresbull.2017.04.052.

[70] M. Schäferling, The art of fluorescence imaging with chemical sensors, Angew. Chem. Int. Ed. 51 (2012) 3532-3554, https://doi.org/10.1002/anie.201105459.

[71] Z. Wang, Z. Fan, Cu2 + modulated nitrogen-doped graphene quantum dots as a turn-off/on fluorescence sensor for the selective detection of histidine in biologica fluid, Spectrochim. Acta A Mol. Biomol. Spectrosc. 189 (2018) 195-201, https:// doi.org/10.1016/j.saa.2017.08.003.

[72] L. Jin, Y. Wang, F. Yan, J. Zhang, F. Zhong, The synthesis and application of nitrogen-doped graphene quantum dots on brilliant blue detection, J. Nanomater. 2019 (2019) 1-9, https://doi.org/10.1155/2019/1471728. 